\title{
A Multi-Phase Theory for the Detonation of Granular Explosives Containing an Arbitrary Number of Solid Components
}

\author{
M. W. Crochet ${ }^{\mathrm{b}, 1, *}$, K. A. Gonthier ${ }^{\mathrm{a}, 2}$ \\ ${ }^{a}$ Department of Mechanical and Industrial Engineering, Louisiana State University, Baton Rouge, LA, USA 70803 \\ ${ }^{b}$ University of Dayton Research Institute, Eglin AFB, Florida, 32542
}

\begin{abstract}
Multiphase continuum models are commonly used to predict the shock, combustion and detonation behavior of granular energetic mixtures containing solid reactants and gaseous products. These models often include phase interaction terms that formally satisfy the strong form of the Second Law of Thermodynamics and provide flexibility in distributing dissipation between phases arising from non-equilibrium phenomena. This work presents a thermodynamically compatible constitutive theory for reactive systems containing an arbitrary number of solid components. The theory represents a rigorous extension of the two-phase theory formulated by Bdzil, et al. [5], based on the well-studied Baer-Nunziato model. Forms of the gas-solid and solid-solid interphase sources suggested by general reactions of type $A \rightarrow B$ are considered, where the combustion processes discussed in [5] are treated as a special case. The model energetics are augmented by supplemental evolutionary equations that track local changes in phase temperatures due to dissipative and transport processes allowing for the identification of dominant energetic processes. This capability provides a means to identify system parameters (e.g., metal particle size and mass fraction in metalized energetic mixtures) which optimize performance metrics. Detonation predictions are given for mixtures of granular HMX and aluminum to demonstrate model features and to highlight the effect of aluminum particle self-heating by oxidation on detonation. Predicted spatial profiles for mechanical fields, and the heating contributions from individual dissipative processes, illustrate how aluminum particle size can affect the the coupling of oxidative heating to the explosive reaction zone.
\end{abstract}

Keywords: continuum modeling, granular media, multiphase mixtures, hyperbolic equations, wave structures

\section{Introduction}

Multiphase models have been used extensively to analyze engineering-scale flow features in a number of scientific disciplines. The modeling of gas-solid flow within fluidized beds has been widely studied in the chemical and petroleum engineering communities because of its relevance to flows in reactors and vertical risers $[43,44,10,27,26]$. Gas flows in porous media have also been examined [45, 20], and reactive dusty gas flows have been investigated because of their potential to detonate in the presence of an ignition source $[23,49,40,22]$. Many of the models used to analyze these systems are based on the Eulerian multiphase continuum approach, where the flow field is represented by overlapping continua which coexist at each spatial location. These models often consist of hyperbolic evolution equations for the quantities of interest, which are supplemented by equations of state and algebraic source terms describing interphase exchange processes.

One of the more influential two-phase Eulerian models is the Baer-Nunziato (BN) system of equations [3], which is used to analyze deflagration-to-detonation transition in granular high explosives (HEs), where the solid HE reacts to form gaseous combustion products. This model consists of compressible, inviscid balance equations for mass, momentum and energy within each phase, coupled with a compaction equation which is used to evolve the solid volume fraction. This model convects the solid volume

\footnotetext{
${ }^{*}$ Corresponding author.

Email addresses: michael.w.crochet@gmail.com (M. W. Crochet), gonthier@me.lsu.edu (K. A. Gonthier)

${ }^{1}$ Computational Researcher, Tel.: +1 850-882-5229

${ }^{2}$ Associate Professor, Tel.: +1 225-578-5915

Preprint submitted to Int. J. Multiphas. Flow

July 19, 2015
}

(C) 2015. This manuscript version is made available under the Elsevier user license

http://www.elsevier.com/open-access/userlicense/1.0/ 


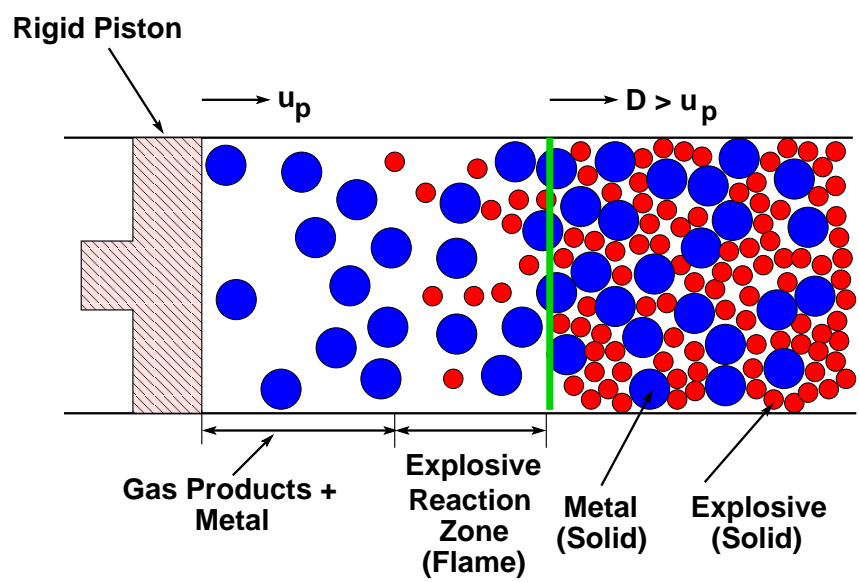

Figure 1: Schematic of Piston-Induced Steady Detonation in a Metalized Explosive

fraction with the local solid velocity, although other authors utilize alternative convection criteria (e.g., mass-averaged velocity) [37, 28]. The forms of the interphase exchange terms were determined using an expression which satisfied the strong form of the solid-gas mixture entropy inequality. The BN model has formed the basis of a large body of subsequent work. The basic approach was expanded by Bdzil et al. (BMSKS) to apportion dissipation to each phase in a physically appealing manner [5]. This is accomplished by introducing additional model parameters, referred to as dissipation partitioning functions, included within the interphase sources. A similar multiphase model capable of analyzing an arbitrary number of phases has also been formulated by Chinnayya et al. [8, 33], which satisfies the mixture entropy inequality; however, it does not explicitly provide the dissipation energy partitioning flexibility of the BMSKS model, which is necessary for a more physically relevant specification of the interphase source terms, nor does it contain a mechanism for explicitly considering solid-gas and solid-solid interactions separately.

An important application of multiphase modeling is the analysis of metalized high explosives. Metal oxidation is widely known to enhance the post-detonation blast wave in explosive charges, and the rate of this oxidation energy release is relatively insensitive to explosive particle size, but strongly dependent on metal particle size [30,41]. Due to their larger surface area-to-volume ratio, it is theorized that the addition of nanometer-sized metal particles may strengthen detonation waves due to more rapid oxidation and the subsequent thermal transport to the explosive combustion gases. However, experimental results measuring the performance of metalized explosives have been inconclusive $[7,24,16,15,19,25]$. Some experiments indicate that mixtures containing metal nanoparticles reduce detonation speeds compared to standard micron-sized particles, while others suggest that detonation speeds can increase under certain conditions [47]. Peak pressures behind the detonation wave have been shown to rise with decreasing metal particle size; furthermore, for a fixed metal particle size, performance may also depend on the type of high explosive used in the mixture. Modeling efforts are therefore an important tool for establishing the dependence of flow properties on mixture parameters.

In many engineering-scale models, detonation in granular energetic materials is typically initiated by mechanical impact due to a rigid piston moving at a speed $u_{p}$, as shown in Fig. 1. In this one-dimensional representation, the reacting explosive component eventually leads to the formation of a steady, planar detonation wave, propagating at a constant speed $D$ into the unreacted mixture. Explosive combustion occurs within a zone of finite thickness behind the detonation front. Behind this region, only the gaseous explosive combustion products and oxidizing metal particles are present.

Figure 2 shows the qualitative wave structure in both the gas-phase pressure $P_{g}$ and the explosive volume fraction $\phi_{e}$ in a piston-attached frame of reference, where the quantities denoted by the " 0 " superscript indicate the initial state and the spatial position relative to the piston surface is denoted by $\xi_{p}$. The gas-phase fields display the qualitative structure shown in Fig. 2(a). Behind the explosive reaction zone is an expansion wave traveling to the right, which relaxes the gas pressure to a constant state in the vicinity of the piston surface. In the explosive phase, the solid is consumed at the end of the explosive reaction zone, as shown in Fig. 2(b). For the simple explosive burn rate model utilized in [39] and adopted here, there are two possible types of steady wave structures: compaction-led, as indicated 


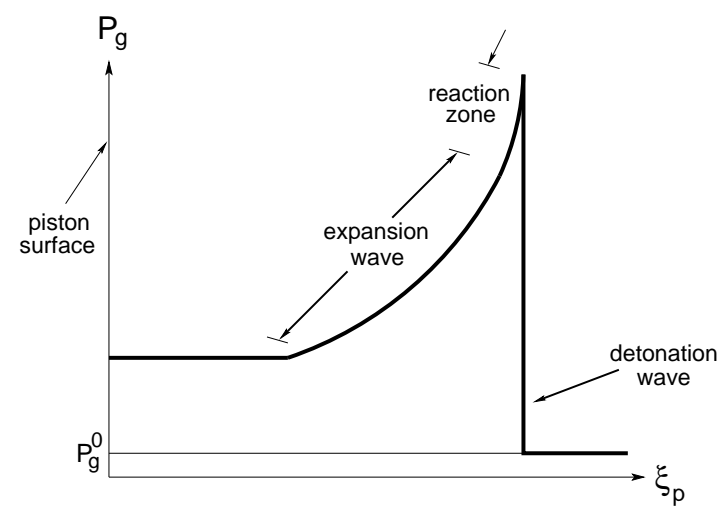

(a)

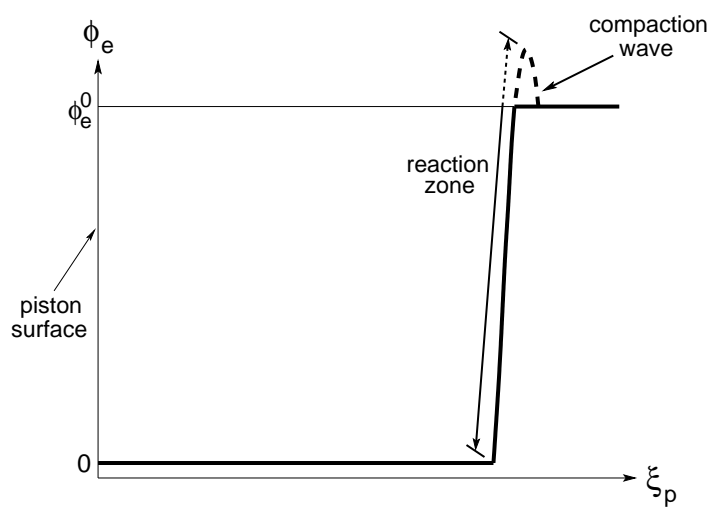

(b)

Figure 2: Qualitative Description of Post-Detonation (a) Gas-Phase Pressure and (b) Explosive Volume Fraction

by the dashed curve in Fig. 2(b), and reaction-led, shown by the constant solid line to the right of the detonation front. Compaction-led detonations are characterized by a compaction wave in the explosive phase to the right of the detonation wave in the gas phase, which results in the formation of a solid plug prior to the passage of the detonation front. For reaction-led detonations the gaseous detonation wave precedes the explosive-phase wave; since explosive combustion occurs immediately behind the detonation front, there is no accumulation of the solid explosive prior to reaction. Here, it is noted that the explosive reaction zone is typically thin, and is magnified in Figs. 1 and 2 for illustration.

Several approaches for modeling detonation in metalized explosives have been proposed in the literature since the introduction of the BN model. Orth-Farrell and Krier provided an analysis of detonation in HMX-aluminum mixtures using a simplified set of mixture evolution equations [31]. Zhang et al. formulated a fully compressible, two-phase model similar to the BN system [48]; however, the model is limited by the simplified constitutive description of the oxidation products. Although it is recognized that metal oxidation is a complex process and proceeds in multiple stages, basic approaches such as Arrhenius-based descriptions [29, 13] or simplified additional evolution equations [21] are highly preferred, due to their simplicity and consistency with the limiting case of vanishing oxidation rate as the reaction proceeds to completion. In particular, Fedorov and Khmel use these constitutive laws in constructing a steady, twophase Eulerian model [14], although their analysis is restricted entirely to the detonation wave structure, and is unable to examine the flow properties in the expansion wave behind the reaction zone; moreover, the steady model has the same limitations as the unsteady models mentioned here in partitioning dissipation energy between phases in a physically plausible manner. Recently, a Lagrangian model with hybrid mixture/multiphase evolution equations was developed by Ruggirello et al. [36] to account for more complex oxidation mechanisms and metal phase change. However, the multiphase component is limited to the BN description, and the numerical strategy for the model implementation within an Eulerian framework is cumbersome due to an additional grid remap step which is not present in standard finite-volume schemes.

The work presented here first makes several modifications to the BMSKS model for an arbitrary number of reactive solid phases. Chemical reaction terms are included in the model, and a rigorous analysis of the mixture entropy inequality is presented, which identifies the physically admissible reaction processes and resulting source terms. This procedure requires the definition of extra dissipation partitioning functions in addition to those given in [5], which are associated with chemical reactions. A framework for developing constitutive relations for the source terms is developed for general chemical reactions of type $A \rightarrow B$ needed for an elementary description of metal oxidation, which is simplified for the special case of exothermic reactions relevant to the combustion of energetic solids. However, this approach can be extended to more complex kinetics. Second, supplemental evolution equations are proposed for a deeper examination of the system energetics. Traditionally, the relative influence of dissipative processes such as drag and compaction are investigated using instantaneous rates [39]. However, these do not account for the unsteady behavior of each dissipative process, nor their cumulative effects over the course of a simulation. The energy balance equations for each phase are therefore modified to evolve changes in temperature due to individual dissipation and transport processes during the simulation, which cumulatively result in the net temperature rise for each phase; these additional relations are referred to as the temperature change decomposition equations for each phase. Using the model formulated here, 
representative results are shown for the steady detonation of aluminized $\operatorname{HMX}\left(\mathrm{C}_{4} \mathrm{H}_{8} \mathrm{~N}_{8} \mathrm{O}_{8}\right)$, initiated by a constant-speed, rigid piston impact.

This paper is organized as follows. Section 2 provides an overview of the reactive multiphase model and use of the mixture entropy inequality to define the source terms. Section 3 gives a discussion of the constitutive relations for the interphase sources, including the formulation of the dissipation partitioning functions for single-stage chemical reactions. Finally, representative one-dimensional results for metalized explosive detonation are given in Section 4, and conclusions are presented in Section 5.

\section{Model Description}

The set of one-dimensional equations is first extended to multiple dimensions and is generalized to account for chemical reactions in Section 2.1. The mixture entropy inequality is then developed in Section 2.2 using expressions for phase entropy evolution. Finally, this basic model is then expanded to include the temperature change decomposition relations in Section 2.3.

\subsection{Balance Laws}

The BN equations applied to a mixture consisting of a gas phase and $M$ solid phases are given by the following equations for a general, three-dimensional formulation:

$$
\frac{\partial \mathbf{q}}{\partial t}+[\nabla \cdot \mathbf{F}(\mathbf{q})]^{\top}=\sum_{j=1}^{M} \mathbf{G}_{j}(\mathbf{q}) \cdot \nabla \phi_{j}^{\top}+\mathbf{s}(\mathbf{q}),
$$

where

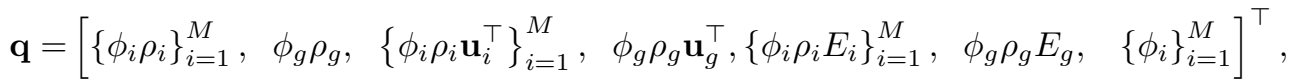

$$
\begin{aligned}
& \mathbf{F}(\mathbf{q})=\left[\left\{\phi_{i} \rho_{i} \mathbf{u}_{i}\right\}_{i=1}^{M}, \quad \phi_{g} \rho_{g} \mathbf{u}_{g}, \quad\left\{\phi_{i}\left(\rho_{i} \mathbf{u}_{i} \mathbf{u}_{i}^{\top}+P_{i} \mathbf{I}\right)\right\}_{i=1}^{M},\right. \\
& \phi_{g}\left(\rho_{g} \mathbf{u}_{g} \mathbf{u}_{g}^{\top}+P_{g} \mathbf{I}\right),\left\{\phi_{i} \rho_{i}\left(E_{i}+P_{i} / \rho_{i}\right) \mathbf{u}_{i}\right\}_{i=1}^{M}, \\
& \left.\phi_{g} \rho_{g}\left(E_{g}+P_{g} / \rho_{g}\right) \mathbf{u}_{g}, \quad\{\mathbf{0}\}_{i=1}^{M}\right] \text {, }
\end{aligned}
$$

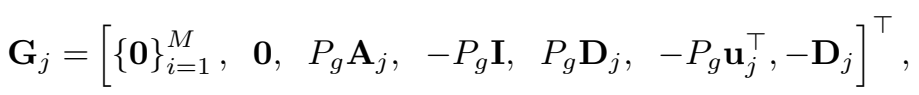

$$
\begin{aligned}
& \mathbf{s}(\mathbf{q})=\left[\left\{\mathcal{C}_{i}\right\}_{i=1}^{M}, \mathcal{C}_{g}, \quad\left\{\overrightarrow{\mathcal{M}}_{i}\right\}_{i=1}^{M}, \quad \overrightarrow{\mathcal{M}_{g}}, \quad\left\{\mathcal{E}_{i}\right\}_{i=1}^{M}, \mathcal{E}_{g}, \quad\left\{\mathcal{F}_{i}+\mathcal{C}_{i} / \rho_{i}\right\}_{i=1}^{M}\right]^{\top}
\end{aligned}
$$

here, $t$ is time; $\phi$ is volume fraction; $\rho$ is mass density; $\mathbf{u}=[u, v, w]^{\top}$ is phase velocity; $P$ is pressure, and $E=e+|\mathbf{u}|^{2} / 2$ is the total mass-specific energy, where $e$ is internal energy. For solid reactant components the internal energy may be expressed generally as the sum of its thermal energy, chemical energy and potential energy associated with granular compaction; for the gaseous product component, its internal energy is simply equal to its thermal energy. These equations contain non-conservative source terms referred to as "nozzling" terms in the literature, consisting of tensors $\mathbf{G}_{j}$ which multiply solid volume fraction gradients, and prevent Eq. (1) from being posed in divergence form. The source terms for interphase mass, momentum and energy balance processes are denoted by $\mathcal{C}, \overrightarrow{\mathcal{M}}$ and $\mathcal{E}$, respectively, and the subscripts $g$ and $i$ denote the gas phase and $i$-th solid phase, respectively, for $i=1,2, \ldots, M$. The $3 \times 3$ identity matrix is given by $\mathbf{I}$; the rank-two, $3 M \times 3$ tensor $\mathbf{A}_{j}$ is represented as a block matrix:

$$
\mathbf{A}_{j}=\left[\mathbf{A}_{j 1}, \mathbf{A}_{j 2}, \ldots, \mathbf{A}_{j M}\right]
$$

where the $3 \times 3$ submatrices

$$
\mathbf{A}_{j k}= \begin{cases}\mathbf{I}, & \text { if } k=j \\ \mathbf{0}, & \text { if } k \neq j\end{cases}
$$

and the $3 \times M$ block matrix $\mathbf{D}_{j}$ is given by:

$$
\mathbf{D}_{j}=\left[\begin{array}{llll}
\mathbf{d}_{j 1}, & \mathbf{d}_{j 2}, \ldots, & \mathbf{d}_{j M}
\end{array}\right],
$$


where the column vectors $\mathbf{d}_{j k}$ are defined by:

$$
\mathbf{d}_{j k}= \begin{cases}\mathbf{u}_{j}^{\top}, & \text { if } k=j \\ \mathbf{0}, & \text { if } k \neq j\end{cases}
$$

for $k=1,2, \ldots, M$. This system is therefore comprised of $3 M$ balance laws for solid mass, momentum and energy, three corresponding relations for the gas phase, and the final $M$ equations represent the compaction law applied to each of the solid phases. Here, it is noted that although the underlying granular microstructure also depends on particle morphology, the only variable available in the multiphase model used to describe this microstructure is $\phi_{i}$. Efforts to include mesoscale physics within the continuum macroscale description have been attempted [5], but require additional submodeling such as mesoscale simulations $[1,2,4,6,32]$, which is beyond the scope of this work.

The gas internal energy is characterized by its thermal equation of state $e_{g}=e_{g}\left(\rho_{g}, P_{g}\right)$, while the solid equation of state includes a volume fraction contribution due to compaction, i.e., $e_{i}=\widehat{e}_{i}\left(\rho_{i}, P_{i}, \phi_{i}\right)$. A detailed discussion of the thermodynamics of this non-classical constitutive description for the solid phase is given in [5]; it is frequently assumed that compaction of the solid phases is an elastic process $[3,5,18]$, and the solid internal energy may be decomposed as the sum of thermal, elastic compaction and chemical energy, i.e., $e_{i}=\widehat{e}_{i}\left(\rho_{i}, P_{i}\right)+B_{i}\left(\phi_{i}\right)+q_{i}$, where $\widehat{e}_{i}$ represents the thermal equation of state for the pure-phase solid, and $B_{i}$ and $q_{i}$ are the compaction potential energy and heat of combustion of the $i$-th phase, respectively. Here, it is assumed that $q_{i}$ is a known constant; consequently, an expression for the compaction potential energy $B_{i}$ must be obtained. This energy is defined as:

$$
B_{i}\left(\phi_{i}\right)=\int_{\phi_{i}^{0}}^{\phi_{i}} \frac{\beta_{i}}{\widetilde{\phi}_{i} \rho_{i}} d \widetilde{\phi}_{i}
$$

where $\phi_{i}^{0}$ and $\beta_{i}$ are the initial solid volume fraction and intergranular stress of the $i$-th phase, respectively. From Eq. (10),

$$
\frac{d B_{i}}{d t_{i}}=\frac{\beta_{i}}{\phi_{i} \rho_{i}} \frac{d \phi_{i}}{d t_{i}}
$$

where the Lagrangian derivative $d / d t_{i}=\partial / \partial t+\mathbf{u}_{i} \cdot \nabla$. Using the compaction equation:

$$
\frac{d \phi_{i}}{d t_{i}}=\mathcal{F}_{i}+\frac{\mathcal{C}_{i}}{\rho_{i}}
$$

an evolution equation for $B_{i}$ is formulated using the continuity equation:

$$
\frac{\partial}{\partial t}\left(\phi_{i} \rho_{i} B_{i}\right)+\nabla \cdot\left(\phi_{i} \rho_{i} \mathbf{u}_{i} B_{i}\right)=\beta_{i}\left(\mathcal{F}_{i}+\frac{\mathcal{C}_{i}}{\rho_{i}}\right)+B_{i} \mathcal{C}_{i} .
$$

The system given by Eqs. (1)-(5) and Eq. (13) is closed using the saturation constraint:

$$
\phi_{g}+\sum_{j=1}^{M} \phi_{j}=1 \text {. }
$$

Furthermore, since mixture mass, momentum and energy are conserved,

$$
\mathcal{C}_{g}+\sum_{j=1}^{M} \mathcal{C}_{j}=0, \quad \overrightarrow{\mathcal{M}}_{g}+\sum_{j=1}^{M} \overrightarrow{\mathcal{M}}_{j}=\mathbf{0}, \quad \mathcal{E}_{g}+\sum_{j=1}^{M} \mathcal{E}_{j}=0
$$

Here, it is also remarked that the BN system of equations presents one of many possible closure models for the mixture mechanics [46]. The simple compaction law (12) used in the BN model is phenomenological, and is a simplification of the grain-scale mechanisms discussed in further detail in [5]. The functional form of the compaction law is also non-unique; recently, a more complex form for $\mathcal{F}_{i}$ was suggested in [38], which includes higher-order microstructure effects. Moreover, the volume fraction $\phi_{i}$ need not advect at its local phase particle velocity $\mathbf{u}_{i}[28,37]$. The model also neglects diffusive processes within each component due to the large time scales associated with these processes (e.g., cook-off) relative to the acoustic scales associated with the propagation of detonation waves. Fully viscous two-phase 
models analogous to the Navier-Stokes equations have been used [34], but this approach may become intractable upon extension to multiple solid components. Due to its wide use in the detonation modeling community $([5,35,39]$ and included references), the BN model represents a reasonable foundation for the construction of a model accounting for mixtures with an arbitrary number of solid components.

For two-phase models with a single solid phase $(M=1)$, a method was proposed by Coquel et al. to transform the solid volume fraction variable $\phi_{s}[9]$, such that its inclusion in the nozzling sources ensures that these quantities vanish when $\phi_{s} \rightarrow 0$. This transformation is applied to the case where $M>1$ in a very straightforward manner, and is given by:

$$
\zeta_{i}=\ln \left(\frac{\phi_{i}}{1-\phi_{i}}\right) \Rightarrow \phi_{i}=\frac{e^{\zeta_{i}}}{1+e^{\zeta_{i}}}
$$

for $i=1,2, \ldots, M$. Since each $\zeta_{i}$ is a function only of $\phi_{i}$,

$$
d \zeta_{i}=\frac{1}{\phi_{i}\left(1-\phi_{i}\right)} d \phi_{i}
$$

The contribution of the $i$-th solid phase to the nozzling sources therefore vanishes as $\phi_{i} \rightarrow 0$. The simple form of the Lagrangian compaction equation is preserved when posed in terms of the transformed volume fractions $\zeta_{i}$ :

$$
\frac{\partial \zeta_{i}}{\partial t}+\mathbf{u}_{i} \cdot \nabla \zeta_{i}=\frac{d \zeta_{i}}{d t_{i}}=\frac{1}{\phi_{i}\left(\zeta_{i}\right)\left[1-\phi_{i}\left(\zeta_{i}\right)\right]}\left(\mathcal{F}_{i}+\frac{\mathcal{C}_{i}}{\rho_{i}}\right)
$$

Therefore, for this system of $N_{e q}=5+7 M$ scalar equations, where $N_{e q}$ is the total number of evolution equations, new nozzling sources $\widehat{\mathbf{G}}_{i}$ can be defined. From Eqs. (17) and (18), all nozzling terms, except those associated with compaction, are multiplied by a factor of $\phi_{i}\left(1-\phi_{i}\right)$. For $i=1,2, \ldots, M$,

$$
\widehat{\mathbf{G}}_{i}(\mathbf{q})=\mathbf{L}_{i} \cdot \mathbf{G}_{i}(\mathbf{q})
$$

where $\mathbf{L}_{i}$ is a $N_{e q} \times 3$ block matrix:

$$
\mathbf{L}_{i}=\left[\begin{array}{llll}
\mathbf{L}_{i 1}, & \mathbf{L}_{i 2}, & \ldots, & \mathbf{L}_{i N_{e q}}
\end{array}\right]^{\top},
$$

where the $j$-th $3 \times 3$ submatrix of $\mathbf{L}_{i}$ is defined by:

$$
\mathbf{L}_{i j}= \begin{cases}\mathbf{I}, & \text { if } j=3 M+3+i \\ \phi_{i}\left(1-\phi_{i}\right) \mathbf{I}, & \text { otherwise }\end{cases}
$$

for $j=1,2, \ldots, N_{e q}$. These modified nozzling sources $\widehat{\mathbf{G}}_{i}(\mathbf{q})$ and the transformed compaction equation (18) may be substituted directly into the system of equations (1)-(5), with the components of $\mathbf{s}(\mathbf{q})$ associated with compaction multiplied by a factor of $\left[\phi_{i}\left(1-\phi_{i}\right)\right]^{-1}$, as shown in the right-hand side of Eq. (18).

\subsection{Mixture Entropy Inequality}

The thermodynamic relationships between phase entropy and other properties are given by:

$$
\begin{gathered}
T_{i} \frac{d \eta_{i}}{d t_{i}}=\frac{d e_{i}}{d t_{i}}-\frac{P_{i}}{\rho_{i}^{2}} \frac{d \rho_{i}}{d t_{i}}-\frac{\beta_{i}}{\phi_{i} \rho_{i}} \frac{d \phi_{i}}{d t_{i}}, \\
T_{g} \frac{d \eta_{g}}{d t_{g}}=\frac{d e_{g}}{d t_{g}}-\frac{P_{g}}{\rho_{g}^{2}} \frac{d \rho_{g}}{d t_{g}} .
\end{gathered}
$$

Equations (22) shows that solid component energy dissipation is generated by changes in internal energy, classical compression work and granular compaction. With the exception of compaction dissipation, Eqn. (23) shows that the same entropy production mechanisms are present in the gaseous component. These equations must now be expressed in terms of the sources $\mathbf{C}_{i}, \overrightarrow{\mathcal{M}}_{i}, \mathcal{E}_{i}$ and $\mathcal{F}_{i}$ to determine the physically admissible restrictions on their algebraic forms. For simplicity the final forms of Eqns. (22) 
and (23) are given here; the details of these derivations are provided in Appendix A.

$$
\begin{aligned}
\phi_{i} \rho_{i} T_{i} \frac{d \eta_{i}}{d t_{i}} & =\mathcal{E}_{i}-\overrightarrow{\mathcal{M}}_{i} \cdot \mathbf{u}_{i}-\mathcal{C}_{i}\left(e_{i}+\frac{\beta_{i}}{\rho_{i}}-\frac{\left|\mathbf{u}_{i}\right|^{2}}{2}\right)+\left(P_{i}-\beta_{i}\right) \mathcal{F}_{i} \\
\phi_{g} \rho_{g} T_{g} \frac{d \eta_{g}}{d t_{g}} & =\mathcal{E}_{g}-\overrightarrow{\mathcal{M}}_{g} \cdot \mathbf{u}_{g}-\mathcal{C}_{g}\left(e_{g}-\frac{\left|\mathbf{u}_{g}\right|^{2}}{2}\right)-\frac{P_{g}}{\rho_{g}} \sum_{j=1}^{M} \mathcal{C}_{j}\left(\frac{\rho_{g}}{\rho_{j}}-1\right) \\
& -P_{g} \sum_{j=1}^{M}\left[\mathcal{F}_{j}-\left(\mathbf{u}_{j}-\mathbf{u}_{g}\right) \cdot \nabla \phi_{j}\right] .
\end{aligned}
$$

The mixture entropy inequality is now formulated. In the absence of thermal conduction, for each phase the Second Law is given by:

$$
\frac{\partial}{\partial t}\left(\phi_{p} \rho_{p} \eta_{p}\right)+\nabla \cdot\left(\phi_{p} \rho_{p} \mathbf{u}_{p} \eta_{p}\right) \geq 0
$$

where $p=i, g$. The continuity equations are used to obtain the Lagrangian form of the mixture entropy inequality:

$$
\phi_{g} \rho_{g} \frac{d \eta_{g}}{d t_{g}}+\sum_{j=1}^{M} \phi_{j} \rho_{j} \frac{d \eta_{j}}{d t_{j}}+\sum_{j=1}^{M} \mathcal{C}_{j}\left(\eta_{j}-\eta_{g}\right) \geq 0 .
$$

Using Eqs. (24) and (25), the mixture entropy inequality expressed in terms of the interphase sources is given by:

$$
\begin{aligned}
& \sum_{j=1}^{M}\left\{\left(\frac{1}{T_{j}}-\frac{1}{T_{g}}\right) \mathcal{E}_{j}-\left(\frac{\mathbf{u}_{j}}{T_{j}}-\frac{\mathbf{u}_{g}}{T_{g}}\right) \cdot \overrightarrow{\mathcal{M}}_{j}+\left(\frac{P_{j}-\beta_{j}}{T_{j}}-\frac{P_{g}}{T_{g}}\right) \mathcal{F}_{j}-\left[( \frac { 1 } { T _ { j } } ) \left(e_{j}+\frac{\beta_{j}}{\rho_{j}}\right.\right.\right. \\
& \left.\left.-\frac{\left|\mathbf{u}_{j}\right|^{2}}{2}-T_{j} \eta_{j}\right)-\left(\frac{1}{T_{g}}\right)\left(e_{g}+\frac{P_{g}}{\rho_{g}}-\frac{\left|\mathbf{u}_{g}\right|^{2}}{2}-T_{g} \eta_{g}\right)\right] \mathcal{C}_{j} \\
& \left.+\frac{P_{g}}{\rho_{g}}\left(\mathbf{u}_{j}-\mathbf{u}_{g}\right) \cdot \nabla \phi_{j}\right\}-\frac{P_{g}}{T_{g}} \sum_{j=1}^{M} \frac{\mathcal{C}_{j}}{\rho_{j}} \geq 0 .
\end{aligned}
$$

The role of Eqn. (28) is central to the construction of the multiphase model, since the interphase source terms must be selected such that the inequality is satisfied exactly throughout the mixture. These source term specifications are the subject of Section 3.

\subsection{Temperature Change Decomposition Equations}

The modeling section is concluded by expanding the system presented in Section 2.1 to include the supplemental relations needed to examine the contributions of each dissipative and transport process to the mixture energetics. For a caloric equation of state $\widehat{e}_{p}\left(\rho_{p}, T_{p}\right)$, where $p=i, g$ and $T$ is the phase temperature, the Chain Rule yields:

$$
d \widehat{e}_{p}=\left(\frac{\partial \widehat{e}_{p}}{\partial \rho_{p}}\right)_{T_{p}} d \rho_{p}+c_{v p} d T_{p}
$$

where $c_{v p}=\left(\partial \widehat{e}_{p} / \partial T_{p}\right)_{\rho_{p}}$ is the mass-specific constant- volume specific heat. From the Gibbs relation,

$$
\phi_{p} \rho_{p} \frac{d \widehat{e}_{p}}{d t_{p}}=\phi_{p} \frac{P_{p}}{\rho_{p}} \frac{d \rho_{p}}{d t_{p}}+\phi_{p} \rho_{p} T_{p} \frac{d \eta_{p}}{d t_{p}} .
$$

Equating the right-hand sides of Eqs. (29) and (30) gives:

$$
\phi_{p} \rho_{p} c_{v p} \frac{d T_{p}}{d t_{p}}=\phi_{p} \rho_{p}\left(\frac{P_{p}}{\rho_{p}^{2}}-\left.\frac{\partial \widehat{e}_{p}}{\partial \rho_{p}}\right|_{T_{p}}\right) \frac{d \rho_{p}}{d t_{p}}+\phi_{p} \rho_{p} T_{p} \frac{d \eta_{p}}{d t_{p}} .
$$

As shown in Eq. (28), the mixture entropy inequality contains heating contributions from several different mechanisms, depending on the forms of the interphase exchange sources. Consequently, the 
total phase temperature change $d T_{p}$ can be decomposed into the sum of several components. The following decomposition is utilized; here, the $p$ subscript is dropped for convenience:

$$
d T=d T_{c p r}+d T_{d r}+d T_{h t}+d T_{c p c}+d T_{c r},
$$

where each term on the right-hand side of Eq. (32) represents a differential change in temperature due to classical compression, interphase drag and heat transfer, compaction and chemical reactions, respectively. For simplicity in initializing simulations, a change of variable is made by subtracting the initial phase temperature $T^{0}$ from each quantity in Eq. (32) and denoting this difference by $\mathcal{T}$ :

$$
d \mathcal{T}=d \mathcal{T}_{c p r}+d \mathcal{T}_{d r}+d \mathcal{T}_{h t}+d \mathcal{T}_{c p c}+d \mathcal{T}_{c r},
$$

where the initial reference state for each component temperature $\mathcal{T}$ is identically zero. Using the continuity equation, this decomposition can be expressed in Eulerian form for inclusion in the system of model equations:

$$
\phi \rho c_{v} \frac{d \mathcal{T}}{d t}=c_{v}\left[\frac{d}{d t}(\phi \rho \mathcal{T})-\mathcal{T} \frac{d}{d t}(\phi \rho)\right]=c_{v}\left[\frac{\partial}{\partial t}(\phi \rho \mathcal{T})+\nabla \cdot(\phi \rho \mathcal{T} \mathbf{u})-\mathcal{C} \mathcal{T}\right]
$$

Therefore, the supplemental evolution equations associated with the temperature change decomposition are given by:

$$
\frac{\partial}{\partial t}\left(\phi_{p} \rho_{p} \overrightarrow{\mathcal{T}}_{p}\right)+\nabla \cdot\left(\phi_{p} \rho_{p} \overrightarrow{\mathcal{T}}_{p} \mathbf{u}_{p}\right)=\mathbf{S}_{p}
$$

where

$$
\begin{gathered}
\overrightarrow{\mathcal{T}}_{p}=\left[\begin{array}{llll}
\mathcal{T}_{c p c, p}, & \mathcal{T}_{d r, p}, & \mathcal{T}_{h t, p}, & \mathcal{T}_{c r, p}
\end{array}\right]^{\top}, \\
\mathbf{S}_{p}=\left[\begin{array}{llll}
S_{c p c, p}, & S_{d r, p}, & S_{h t, p}, & S_{c r, p}
\end{array}\right]^{\top},
\end{gathered}
$$

and the source terms in $\mathbf{S}_{p}$ are associated with each corresponding energetic process. Because the compression contribution cannot be expressed as an explicit algebraic function due to the presence of $\nabla \cdot \mathbf{u}_{p}$ in $d \rho_{p} / d t_{p}$, this term is not evolved separately in Eq. (36). Instead, the energy balance equation is first used to evolve phase temperatures through the use of the equation of state. The temperature change decomposition contributions in Eq. (36) are then subtracted from the evolved phase temperature difference to obtain the compression contribution. The forms of the sources in Eq. (36) are determined by the specifications of the interphase exchange terms $\overrightarrow{\mathcal{M}}, \mathcal{E}$ and $\mathcal{F}$, which are discussed in the following section.

It should also be noted that the selection of the temperature change decomposition need not be unique. For instance, a particular dissipative process for a single solid phase could be further decomposed into separate solid-solid and solid-gas contributions. However, computational tractability may be compromised, since this choice would increase the total number of model equations from $O(M)$ to $O\left(M^{2}\right)$, and such decompositions are therefore not considered here.

\subsection{One-Dimensional Model Review}

For the simulations performed in this work, the final set of one-dimensional model equations is given by:

$$
\frac{\partial \mathbf{q}}{\partial t}+\frac{\partial \mathbf{f}(\mathbf{q})}{\partial x}=\sum_{j=1}^{M} \mathbf{g}_{j}(\mathbf{q}) \frac{\partial \phi_{j}}{\partial x}+\mathbf{s}(\mathbf{q}),
$$

where

$$
\begin{aligned}
& \mathbf{q}=\left[\left\{\phi_{i} \rho_{i}\right\}_{i=1}^{M}, \quad \phi_{g} \rho_{g}, \quad\left\{\phi_{i} \rho_{i} u_{i}\right\}_{i=1}^{M}, \phi_{g} \rho_{g} u_{g},\left\{\phi_{i} \rho_{i} E_{i}\right\}_{i=1}^{M}, \phi_{g} \rho_{g} E_{g},\left\{\phi_{i}\right\}_{i=1}^{M}, \quad\left\{\phi_{i} \rho_{i} B_{i}\right\}_{i=1}^{M},\right. \\
& \left.\left\{\phi_{i} \rho_{i} \overrightarrow{\mathcal{T}}_{i}\right\}_{i=1}^{M}, \phi_{g} \rho_{g} \overrightarrow{\mathcal{T}}_{g}\right]^{\top} \\
& \mathbf{f}(\mathbf{q})=\left[\left\{\phi_{i} \rho_{i} u_{i}\right\}_{i=1}^{M}, \quad \phi_{g} \rho_{g} u_{g}, \quad\left\{\phi_{i} \rho_{i} u_{i}^{2}+\phi_{i} P_{i}\right\}_{i=1}^{M}, \phi_{g} \rho_{g} u_{g}^{2}+\phi_{g} P_{g}, \quad\left\{\phi_{i} \rho_{i} u_{i}\left(E_{i}+P_{i} / \rho_{i}\right)\right\}_{i=1}^{M},\right. \\
& \left.\phi_{g} \rho_{g} u_{g}\left(E_{g}+P_{g} / \rho_{g}\right), \quad\{0\}_{i=1}^{M}, \quad\left\{\phi_{i} \rho_{i} u_{i} B_{i}\right\}_{i=1}^{M},\left\{\phi_{i} \rho_{i} u_{i} \overrightarrow{\mathcal{T}}_{i}\right\}_{i=1}^{M}, \phi_{g} \rho_{g} u_{g} \overrightarrow{\mathcal{T}}_{g}\right]^{\top},
\end{aligned}
$$




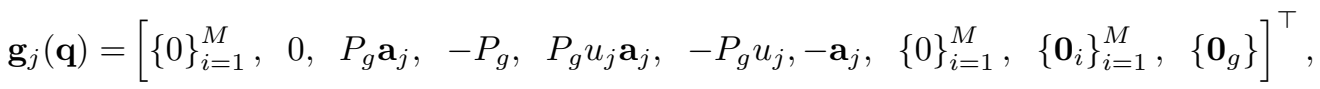

$$
\begin{aligned}
& \mathbf{s}(\mathbf{q})=\left[\left\{\mathcal{C}_{i}\right\}_{i=1}^{M}, \mathcal{C}_{g},\{\mathcal{M}\}_{i=1}^{M}, \mathcal{M}_{g},\left\{\mathcal{E}_{i}\right\}_{i=1}^{M}, \mathcal{E}_{g},\right. \\
& \left.\left\{\mathcal{F}_{i}+\mathcal{C}_{i} / \rho_{i}\right\}_{i=1}^{M}, \quad\left\{\beta_{i}\left(\mathcal{F}_{i}+\mathcal{C}_{i} / \rho_{i}\right)+B_{i} \mathcal{C}_{i}\right\}_{i=1}^{M},\left\{\mathbf{S}_{i}\right\}_{i=1}^{M}, \mathbf{S}_{g}\right]^{\top},
\end{aligned}
$$

where the $\mathbf{a}_{j}$ 's are vectors of length $M$ such that the $k$-th component $a_{j k}$ is given by:

$$
a_{j k}= \begin{cases}0, & \text { if } k \neq j \\ 1, & \text { if } k=j\end{cases}
$$

for $k=1,2, \ldots, M$, and $\mathcal{M}$ is the one-dimensional analogue of $\overrightarrow{\mathcal{M}}$. The system given by Eq. (37) is solved numerically using a finite-volume numerical method described in [12] to obtain the predictions presented here.

\section{Interphase Exchange Terms}

The model equations include mass, momentum and energy exchanges between all $M+1$ phases, indicated by $\mathcal{C}, \overrightarrow{\mathcal{M}}$ and $\mathcal{E}$, respectively. These interactions may occur between solid and gas phases, or between any two distinct solid phases. For instance, the combustion of a high explosive is often modeled at the macroscale such that the reaction results in the formation of a gas phase only, whereas metal oxidation within the same gas can produce a separate metal oxide phase. Here, the mixture entropy inequality Eq. 28) is used to suggest functional forms for each source term consistent with the strong form of the Second Law. The resulting forms are not unique, but characterize the interphase transport processes in terms of parameters that may be obtained from empirical studies or mesoscale submodels.

\subsection{Solid-Gas and Solid-Solid Interactions}

These sources are decomposed using the method described in [18] for one-dimensional, non-reactive systems:

$$
\mathcal{C}_{i}=\mathcal{C}_{i g}+\sum_{n=1}^{M} \mathcal{C}_{i n}, \quad \overrightarrow{\mathcal{M}}_{i}=\overrightarrow{\mathcal{M}}_{i g}+\sum_{n=1}^{M} \overrightarrow{\mathcal{M}}_{i n}, \quad \mathcal{E}_{i}=\mathcal{E}_{i g}+\sum_{n=1}^{M} \mathcal{E}_{i n}, \quad \mathcal{F}_{i}=\mathcal{F}_{i g}+\sum_{n=1}^{M} \mathcal{F}_{i n}
$$

where the subscript $i g$ denotes an exchange between the $i$-th solid phase and the gas phase, and the subscript $i n$ indicates an exchange between the $i$-th and $n$-th solid phases. Since there are no interactions between a solid phase and itself, it follows that if $i=n$, then $\mathcal{W}_{i n}=0$, where $\mathcal{W}_{i n}$ represents any of the solid-solid mass, momentum, energy, or compaction source terms. These interactions must also be equal and opposite, i.e., $\mathcal{W}_{i n}=-\mathcal{W}_{n i}$. Therefore,

$$
\sum_{j=1}^{M} \sum_{n=1}^{M} \mathcal{W}_{j n}=0
$$

Consequently, when the phase entropy inequalities (22) and (23) are expanded using these decompositions, the resulting equations include terms having the form $\left(z_{i} / T_{i}-z_{g} / T_{g}\right)$ and $\left(z_{i} / T_{i}-z_{n} / T_{n}\right)$, where $z_{i}, z_{g}$ and $z_{n}$ can be arbitrary scalar or vector quantities appearing in the inequalities. This expansion thus allows the mixture relation 28 to be posed in terms of the solid-gas and solid-solid interactions. The details of these derivations may be found in Appendix B.

\subsection{Partitioning Functions}

The strong form of the Second Law may be identically satisfied by selecting $\mathcal{C}, \overrightarrow{\mathcal{M}}, \mathcal{E}$ and $\mathcal{F}$ such that Eq. (28) is expressed as a sum of squares. From the one-dimensional theory, it is recognized in [5] that, for arbitrary quantities $y_{i}, y_{g}, z_{i}$ and $z_{n}$,

$$
\left(\frac{y_{i}}{T_{i}}-\frac{y_{g}}{T_{g}}\right)=\left(\frac{1}{T_{i}}-\frac{1}{T_{g}}\right)\left[y_{i} w_{i g}+y_{g}\left(1-w_{i g}\right)\right]+\left(y_{i}-y_{g}\right)\left(\frac{1-w_{i g}}{T_{i}}+\frac{w_{i g}}{T_{g}}\right),
$$




$$
\left(\frac{z_{i}}{T_{i}}-\frac{z_{n}}{T_{n}}\right)=\left(\frac{1}{T_{i}}-\frac{1}{T_{n}}\right)\left[z_{i} w_{i n}+z_{m}\left(1-w_{i n}\right)\right]+\left(z_{i}-z_{n}\right)\left(\frac{1-w_{i n}}{T_{i}}+\frac{w_{i n}}{T_{n}}\right),
$$

for $i, n=1,2, \ldots, M$, where $w_{i g}$ and $w_{i n}$ are scalar weighting functions used to apportion energy dissipation between the phases in solid-gas and solid-solid interactions, respectively. For the latter, it follows that energetic processes between solid phases are equal and opposite, i.e., $w_{i n}=-w_{n i}$. As stated in [5], there are multiple choices for these weighting, or partitioning, functions.

Since an arbitrary number of solid phases is considered, and the model is not restricted to combustion reactions, it is not necessary that $\mathcal{C}_{i}<0$ or $\mathcal{C}_{i g}<0$ in general. In addition, since $\mathcal{C}_{n i}=-\mathcal{C}_{i n}$, there exist non-negative values of $\mathcal{C}_{i n}$; consequently, the assumptions and subsequent chemical reaction partitioning functions used in [5] are not necessarily valid for the systems considered in this work. For these reasons chemical reactions are classified here according to two types: "general" reactions for which any of the above conditions are satisfied, and "combustion" reactions where $\mathcal{C}_{i g}<0$ and $\mathcal{C}_{i n} \equiv 0$.

\subsubsection{General Reactions}

From a modeling perspective, the primary difference between general and combustion reactions is treatment of the phase entropy values, and therefore the selections of $y$ and $z$ in Eqs. (45) and (46) that multiply the mass sources $\mathcal{C}$. Here, no assumptions regarding the values of $\eta_{i}$ and $\eta_{g}$ are made a priori, and these choices are evident from Eqs. (B.3)-(B.5):

$$
y_{i}=e_{i}+\frac{\beta_{i}}{\rho_{i}}-T_{i} \eta_{i}, \quad y_{g}=e_{g}+P_{g}\left(\frac{1}{\rho_{g}}-\frac{1}{\rho_{i}}\right)-T_{g} \eta_{g}
$$

Several partitioning functions are defined using the expansions in Eqs. (45) and (46), and the strong form of the mixture entropy inequality is satisfied by requiring each term in Eq. (B.3) to be non-negative, and

solving for the corresponding value of $\overrightarrow{\mathcal{M}}, \mathcal{E}$ or $\mathcal{F}$. The necessary algebra is straightforward, but lengthy and tedious; for brevity, the final forms of the interphase source terms obtained from this procedure are presented here. A full discussion of this process and derivation of these quantities may be found in [11], which also accounts for additional modeling considerations in the selection of partitioning functions, such as Galilean invariance. The sources are given by:

$$
\begin{gathered}
\mathcal{F}_{i g}=\frac{1}{\mu_{i g}} \phi_{i} \phi_{g}\left(P_{i}-\beta_{i}-P_{g}\right) \\
\overrightarrow{\mathcal{M}}_{i g}=P_{g} \nabla \phi_{i}+\left[\frac{1}{2}\left(\mathbf{u}_{i}+\mathbf{u}_{g}\right)-\alpha_{i g}\left(\mathbf{u}_{g}-\mathbf{u}_{i}\right)\right] \mathcal{C}_{i g}-\delta_{i g}\left(\mathbf{u}_{i}-\mathbf{u}_{g}\right) \\
\mathcal{E}_{i g}=\mathbf{u}_{i} \cdot \overrightarrow{\mathcal{M}}_{i g}+a_{i g} \delta_{i g}\left|\mathbf{u}_{i}-\mathbf{u}_{g}\right|^{2}-\frac{1}{2} \alpha_{i g}\left|\mathbf{u}_{i}-\mathbf{u}_{g}\right|^{2} \mathcal{C}_{i g} \\
-\frac{\left|\mathbf{u}_{i}\right|^{2}}{2} \mathcal{C}_{i g}-\left[c_{i g}\left(P_{i}-\beta_{i}\right)+\left(1-c_{i g}\right) P_{g}\right] \mathcal{F}_{i g} \\
+\mathcal{H}_{i g}\left(T_{g}-T_{i}\right)+\left\{\xi_{i g}\left(e_{i}+\frac{\beta_{i}}{\rho_{i}}-T_{i} \eta_{i}\right)\right. \\
\left.+\left(1-\xi_{i g}\right)\left[e_{g}+P_{g}\left(\frac{1}{\rho_{g}}-\frac{1}{\rho_{i}}\right)-T_{g} \eta_{g}\right]\right\} \mathcal{C}_{i g} \\
\mathcal{F}_{i n}=\frac{1}{\mu_{i n}} \phi_{i} \phi_{m}\left[\left(P_{i}-\beta_{i}\right)-\left(P_{n}-\beta_{n}\right)\right] \\
\mathcal{E}_{i n}=\mathbf{u}_{i} \cdot \overrightarrow{\mathcal{M}}_{i n}-\frac{1}{2} \alpha_{i n}\left|\mathbf{u}_{i}-\mathbf{u}_{n}\right|^{2} \mathcal{C}_{i n}-\frac{\left|\mathbf{u}_{i}\right|^{2}}{2} \mathcal{C}_{i n}+a_{i n} \delta_{i n}\left|\mathbf{u}_{i}-\mathbf{u}_{n}\right|^{2}+\mathcal{H}_{i n}\left(T_{n}-T_{i}\right) \\
-\left[c_{i n}\left(P_{i}-\beta_{i}\right)+\left(1-c_{i n}\right)\left(P_{n}-\beta_{n}\right)\right] \mathcal{F}_{i n} \\
+\left\{\xi_{i n}\left[e_{i}+\frac{\beta_{i}+P_{g} T_{i} / T_{g}}{\rho_{i}}-T_{i} \eta_{i}\right]+\left(1-\xi_{i n}\right)\left[e_{n}+\frac{\beta_{n}+P_{g} T_{n} / T_{g}}{\rho_{n}}-T_{n} \eta_{n}\right]\right\} \mathcal{C}_{i n}
\end{gathered}
$$

Here, the quantities $\mu, \delta$ and $H$ are compaction viscosity, drag and heat transfer coefficients which govern the rate of pressure, velocity and temperature equilibration between the phases, respectively. 
The quantities $a$ and $c$ are dissipation partitioning functions for drag and compaction processes which do not involve mass transfer, respectively; and the partitioning functions $\xi$ and $\alpha$ are associated with dissipation due to chemical reactions.

Additionally, since $\mathcal{C}_{i g}$ and $\mathcal{C}_{i n}$ are specified functions, the strong form is only satisfied if the following restrictions on the $\xi$ and $\alpha$ partitioning functions are imposed:

$$
\begin{gathered}
\sum_{j=1}^{M}\left[\left(e_{g}-e_{j}\right)+\frac{P_{g}}{\rho_{g}}-\frac{P_{g}+\beta_{j}}{\rho_{j}}+T_{j} \eta_{j}-T_{g} \eta_{g}\right]\left(\frac{1-\xi_{j g}}{T_{j}}+\frac{\xi_{j g}}{T_{g}}\right) \mathcal{C}_{j g} \\
+\sum_{j=1}^{M} \sum_{n=j+1}^{M}\left[\left(e_{n}-e_{j}\right)+\frac{\beta_{n}+P_{g} T_{n} / T_{g}}{\rho_{n}}-\frac{\beta_{j}+P_{g} T_{j} / T_{g}}{\rho_{j}}\right. \\
\left.+T_{j} \eta_{j}-T_{n} \eta_{n}\right]\left(\frac{1-\xi_{j n}}{T_{j}}+\frac{\xi_{j n}}{T_{n}}\right) \mathcal{C}_{j n} \geq 0 \\
\sum_{j=1}^{M}\left[\left(\frac{1}{T_{j}}+\frac{1}{T_{g}}\right) \alpha_{j g}\left|\mathbf{u}_{g}-\mathbf{u}_{i}\right|^{2} \mathcal{C}_{i g}\right]+\sum_{j=1}^{M-1} \sum_{n=j+1}^{M}\left[\left(\frac{1}{T_{j}}+\frac{1}{T_{n}}\right) \alpha_{j n}\left|\mathbf{u}_{n}-\mathbf{u}_{j}\right|^{2} \mathcal{C}_{j n}\right] \leq 0
\end{gathered}
$$

It is noted that similar restrictions are not required in [5] for combustion-only reactions. Furthermore, the product $\phi_{i} \phi_{g}$ is introduced in the definition of $\mathcal{F}_{i}$ to guarantee that $\mathcal{F}_{i} \rightarrow 0$ in the limit of vanishing phases. This practice is followed in the literature [3,39], and also ensures that the volume fractions remain positive as the compaction equation is evolved. This is particularly important for numerical stability for combustion processes where $\phi_{i} \rightarrow 0$.

\subsubsection{Combustion Reactions}

When the analysis is confined to the special case of solid phases which undergo only combustion reactions, the selection of dissipation partitioning functions becomes simpler and less restrictive. Because $\mathcal{C}_{i n} \equiv 0$ for all $i$ and $n$, only the $y$ terms in Eq. (45) need to be selected to perform the decomposition. By taking

$$
y_{i}=e_{i}, \quad y_{g}=e_{g}+P_{g}\left(\frac{1}{\rho_{g}}-\frac{1}{\rho_{i}}\right),
$$

the mixture entropy inequality given by Eq. (28) suggests the following forms for the interphase exchange sources; full details of the derivation are again provided in [11]. Letting $\mathcal{C}_{i g}=\mathcal{C}_{i}$,

$$
\begin{gathered}
\mathcal{F}_{i g}=\frac{1}{\mu_{i g}} \phi_{i} \phi_{g}\left(P_{i}-\beta_{i}-P_{g}\right) \\
\overrightarrow{\mathcal{M}}_{i g}=P_{g} \nabla \phi_{i}+\left[\frac{1}{2}\left(\mathbf{u}_{i}+\mathbf{u}_{g}\right)-\alpha_{i}\left(\mathbf{u}_{g}-\mathbf{u}_{i}\right)\right] \mathcal{C}_{i}-\delta_{i g}\left(\mathbf{u}_{i}-\mathbf{u}_{g}\right) \\
\mathcal{E}_{i g}=\mathbf{u}_{i} \cdot \overrightarrow{\mathcal{M}}_{i g}-\left[c_{i g}\left(P_{i}-\beta_{i}\right)+\left(1-c_{i g}\right) P_{g}\right] \mathcal{F}_{i g}-\left[\frac{1}{2} \alpha_{i}\left|\mathbf{u}_{i}-\mathbf{u}_{g}\right|^{2}+\frac{\left|\mathbf{u}_{i}\right|^{2}}{2}\right] \mathcal{C}_{i}+a_{i g} \delta_{i g}\left|\mathbf{u}_{i}-\mathbf{u}_{g}\right|^{2} \\
+\left\{\xi_{i} e_{i}+\left(1-\xi_{i}\right)\left[e_{g}+P_{g}\left(\frac{1}{\rho_{g}}-\frac{1}{\rho_{i}}\right)\right]\right\} \mathcal{C}_{i}+\mathcal{H}_{i g}\left(T_{g}-T_{i}\right), \\
\mathcal{F}_{i n}=\frac{1}{\mu_{i n}} \phi_{i} \phi_{m}\left[\left(P_{i}-\beta_{i}\right)-\left(P_{n}-\beta_{n}\right)\right] \\
\overrightarrow{\mathcal{M}}_{i n}=-\delta_{i n}\left(\mathbf{u}_{i}-\mathbf{u}_{n}\right), \\
\mathcal{E}_{i n}=\left[b_{i n} \mathbf{u}_{i}+\left(1-b_{i n}\right) \mathbf{u}_{n}\right] \cdot \overrightarrow{\mathcal{M}}_{i n}+\mathcal{H}_{i n}\left(T_{n}-T_{i}\right) \\
-\left[c_{i n}\left(P_{i}-\beta_{i}\right)+\left(1-c_{i n}\right)\left(P_{n}-\beta_{n}\right)\right] \mathcal{F}_{i n} .
\end{gathered}
$$

Here, $b_{i n}$ is a solid-solid drag dissipation partitioning function; since all chemical reactions occur between the solid phases and the gas, $\alpha_{i}$ and $\xi_{i}$ do not contain a second subscript to indicate the absence of solidsolid reaction energy dissipation. 
From the choices of $y$, the mixture dissipation inequality is only satisfied if

$$
\sum_{j=1}^{M}\left\{\left[e_{j}-e_{g}-P_{g}\left(\frac{1}{\rho_{g}}-\frac{1}{\rho_{j}}\right)\right]\left(\frac{1-\xi_{j}}{T_{j}}+\frac{\xi_{j}}{T_{g}}\right) \mathcal{C}_{j}+\left[\eta_{g}-\eta_{j}+\frac{\beta_{j}}{\rho_{j} T_{j}}\right] \mathcal{C}_{j}\right\} \leq 0 .
$$

Thus, if Eq. (63) is valid, then there are no physical conditions which must be imposed upon the selection of $\alpha_{i}$ by the Second Law. In [5], the authors argue that for combustion reactions, $\eta_{g}>\eta_{i}, T_{g}>T_{i}$ and $e_{g}-e_{i}+P_{g}\left(\rho_{g}^{-1}-\rho_{i}^{-1}\right)>0$; consequently,

$$
\left[e_{g}-e_{i}+P_{g}\left(\frac{1}{\rho_{g}}-\frac{1}{\rho_{i}}\right)\right]\left(\frac{1-\xi_{i}}{T_{i}}+\frac{\xi_{i}}{T_{g}}\right) \leq \eta_{g}-\eta_{i}+\frac{\beta_{i}}{\rho_{i} T_{i}}
$$

for many physically plausible values of $\xi$ used in [3] and [5]. Satisfying this condition for each individual solid phase for $i=1,2, \ldots, M$ therefore identically satisfies Eq. (63).

From this analysis it is evident that conditions on the use of $\xi_{i}$ and $\alpha_{i}$ are significantly more relaxed in the special case of combustion reactions only compared to more general reactions. This choice for the interphase exchange terms for combustion reactions also has another significant advantage over the general reaction formulation, which is that phase entropy does not need to be evaluated explicitly. Depending on the equation of state considered, the phase entropy values may be difficult to compute, or even unavailable if the equation of state is obtained in tabular form from experimental data.

\subsection{Temperature Change Decomposition Sources}

After obtaining expressions for $\overrightarrow{\mathcal{M}}, \mathcal{E}$ and $\mathcal{F}$ in the previous sections, the phase entropy evolution equations (B.6) and (B.7) can be fully evaluated, and are used to obtain the sources for the temperature change decomposition evolution equations discussed in Section 2.3. Here, it should be noted that the expressions for drag and chemical reaction sources depend on the forms of the interphase exchange sources arising from general and combustion-only reactions.

Evaluating the right-hand side of Eq. (31) for each component of the temperature change decomposition yields:

$$
\begin{gathered}
S_{c p c, i}=\mathcal{C}_{i} \mathcal{T}_{c p c, i}+\frac{1}{c_{v i}}\left\{\frac{1}{\mu_{i g}} \phi_{i} \phi_{g}\left(1-c_{i g}\right)\left(P_{i}-\beta_{i}-P_{g}\right)^{2}\right. \\
\left.+\sum_{n=1}^{M}\left[\frac{1}{\mu_{i n}}\left(1-c_{i n}\right)\left[\left(P_{i}-\beta_{i}\right)-\left(P_{n}-\beta_{n}\right)\right]^{2}\right]\right\} \\
S_{c p c, g}=\mathcal{C}_{g} \mathcal{T}_{c p c, g}+\frac{1}{c_{v g}}\left[\sum_{j=1}^{M} \frac{1}{\mu_{j g}} \phi_{j} \phi_{g} c_{j g}\left(P_{j}-\beta_{j}-P_{g}\right)^{2}\right], \\
S_{d r, g}=\mathcal{C}_{g} \mathcal{T}_{d r, g}+\frac{1}{c_{v g}}\left[\sum_{j=1}^{M} \delta_{j g}\left(1-a_{j g}\right)\left|\mathbf{u}_{i}-\mathbf{u}_{g}\right|^{2}\right] \\
S_{h t, i}=\frac{1}{c_{v i}}\left\{\mathcal{H}_{i g}\left(T_{g}-T_{i}\right)+\sum_{n=1}^{M}\left[\mathcal{H}_{i n}\left(T_{n}-T_{i}\right)\right]\right\}+\mathcal{C}_{i} \mathcal{T}_{h t, i}, \\
S_{h t, g}=\mathcal{C}_{g} \mathcal{T}_{d r, g}+\frac{1}{c_{v g}}\left[-\sum_{j=1}^{M} H_{j g}\left(T_{g}-T_{j}\right)\right]
\end{gathered}
$$

General reactions:

$$
S_{d r, i}=\frac{1}{c_{v i}}\left[\delta_{i g} a_{i g}\left|\mathbf{u}_{i}-\mathbf{u}_{g}\right|^{2}+\sum_{n=1}^{M}\left(\delta_{i n} a_{i n}\left|\mathbf{u}_{i}-\mathbf{u}_{n}\right|^{2}\right)\right]+\mathcal{C}_{i} \mathcal{T}_{d r, i},
$$




$$
\begin{aligned}
& S_{c r, i}=\frac{1}{c_{v i}}\left\{\left[\left(1-\xi_{i g}\right)\left(e_{g}-e_{i}\right)-\left(1-\xi_{i g}\right)\left(T_{g} \eta_{g}\right)-\xi_{i g}\left(T_{i} \eta_{i}\right)-\frac{1}{2} \alpha_{i g}\left|\mathbf{u}_{i}-\mathbf{u}_{g}\right|^{2}\right] \mathcal{C}_{i g}\right. \\
&\left.+\sum_{n=1}^{M}\left[\left(1-\xi_{i n}\right)\left(e_{n}-e_{i}\right)-\xi_{i n}\left(T_{i} \eta_{i}\right)-\left(1-\xi_{i n}\right)\left(T_{n} \eta_{n}\right)\right] \mathcal{C}_{i n}\right\}+\mathcal{C}_{i} \mathcal{T}_{c r, i}, \\
& S_{c r, g}=\frac{1}{c_{v g}}\left\{\sum_{j=1}^{M}\left[\xi_{i g}\left(e_{g}-e_{i}\right)+\xi_{i g}\left(T_{i} \eta_{i}\right)+\left(1-\xi_{i g}\right)\left(T_{g} \eta_{g}\right)+\frac{1}{2} \alpha_{i g}\left|\mathbf{u}_{i}-\mathbf{u}_{g}\right|^{2}\right]\right\}+\mathcal{C}_{g} \mathcal{T}_{c r, g},
\end{aligned}
$$

Combustion reactions only:

$$
\begin{gathered}
S_{d r, i}=\frac{1}{c_{v i}}\left\{\delta_{i g} a_{i g}\left|\mathbf{u}_{i}-\mathbf{u}_{g}\right|^{2}+\sum_{n=1}^{M}\left[\delta_{i n}\left(1-b_{i n}\right)\left|\mathbf{u}_{i}-\mathbf{u}_{n}\right|^{2}\right]\right\}+\mathcal{C}_{i} \mathcal{T}_{d r, i} \\
S_{c r, i}=\mathcal{C}_{i} \mathcal{T}_{c r, i}+\frac{1}{c_{v i}}\left[\left(1-\xi_{i}\right)\left(e_{g}-e_{i}\right)-\frac{1}{2} \alpha_{i}\left|\mathbf{u}_{i}-\mathbf{u}_{g}\right|^{2}\right] \mathcal{C}_{i}, \\
S_{c r, g}=\frac{1}{c_{v g}}\left\{\sum_{j=1}^{M}\left[\xi_{j}\left(e_{g}-e_{j}\right)+\frac{1}{2} \alpha_{j}\left|\mathbf{u}_{j}-\mathbf{u}_{g}\right|^{2}\right] \mathcal{C}_{j}\right\}+\mathcal{C}_{g} \mathcal{T}_{c r, g} .
\end{gathered}
$$

\section{Representative Metalized Explosive Simulations}

In this work results are shown for an energetic mixture with properties that are representative of aluminized HMX; the mixture therefore consists of an explosive phase $(e)$, metal phase $(m)$, and gas phase $(g)$. Piston speed and initial packing density are held constant, and both the explosive and metal phases each have a distinct, uniform particle size. Results are obtained for both inert and oxidizing aluminum; for the latter, a simple empirical relation describing the chemical kinetics provided in [13] is used here. A simple modification to the set of model equations formulated in Section 2.1 is utilized here to account for reactive metal, and an additional contribution to the metal temperature change decomposition is also formulated. It must be emphasized that the results shown here do not constitute a detailed parametric study, which is beyond the scope of this work. Rather, the intent is to illustrate the application of the present model to metalized high explosive mixtures, and how the model can facilitate a more detailed analysis of the system energetics than the conventional use of instantaneous heating rates.

An explicit, second-order centered finite-volume method was used to solve the governing equations numerically. This semidiscrete scheme solves a system of ordinary differential equations within each computational cell, and utilized an operator splitting technique to integrate the nozzling sources in a convective step, followed by integration of the local interphase sources. Details of this method and its formulation are given by Crochet and Gonthier [12]. Simulations were performed with a constant piston speed $u_{p}=100 \mathrm{~m} / \mathrm{s}$, with 2000 computational cells. A spatial domain of $50 \mathrm{~cm}$ was used, and solutions were obtained at time $t=55 \mu$ s to ensure the development of a steady detonation. The mixture is initially at rest relative to the laboratory frame of reference, and is in thermal and mechanical equilibrium, i.e., $P_{e}^{0}=P_{m}^{0}=P_{g}^{0}=P^{0}$ and $T_{e}^{0}=T_{m}^{0}=T_{g}^{0}=T^{0}$, where the " 0 " superscript denotes the initial state. A constant HMX particle size $d_{e}=100 \mu \mathrm{m}$ is used; the total solid volume fraction $\phi_{s}^{0}=95 \%$ is held constant, representing an initially packed mixture. For specified initial values of the metal mass fraction $\lambda_{m}^{0}$, the initial values of $\phi_{e}^{0}$ and $\phi_{m}^{0}$ are obtained after some algebraic manipulation:

$$
\phi_{m}^{0}=\frac{\phi_{s}^{0} \rho_{e}^{0}+\left(1-\phi_{s}^{0}\right) \rho_{g}^{0}}{\rho_{m}^{0}\left(\frac{1-\lambda_{m}^{0}}{\lambda_{m}^{0}}\right)+\rho_{e}^{0}}, \quad \phi_{e}^{0}=\phi_{s}^{0}-\phi_{m}^{0} .
$$

A full discussion of the equations of state and selection of relaxation parameters and dissipation partitioning functions is given in [11]. However, the explosive burn rate and equations of state are provided here for completeness.

For the explosive phase, the simple pressure-dependent burn rate model used by Schwendeman et al. 
in [39] is adopted here:

$$
\mathcal{C}_{e g}= \begin{cases}-\sigma_{e g} \phi_{e} \rho_{e}\left(\frac{P_{g}-P_{i g n, e}}{P_{d c, e}}\right), & P_{g}>P_{\text {ign }, e} \\ 0, & \text { otherwise }\end{cases}
$$

where $\sigma_{e g}$ is a reaction prefactor, and $P_{i g n, e}$ and $P_{d c, e}$ are constant parameters. The explosive phase is assumed to obey a stiffened equation of state:

$$
\widehat{e}_{e}=\frac{P_{e}+\gamma_{e} P_{s t, e}}{\left(\gamma_{e}-1\right) \rho_{e}}
$$

with parameters given in [39]. The metal phase is modeled with a Mie-Grüneisen equation of state. Here, the Grüneisen coefficient $G_{m}$ is approximated by:

$$
G_{m}=\Gamma_{m}\left(\frac{v_{m}}{v_{m}^{*}}\right)^{m_{m}}
$$

where $\gamma$ is the ratio of specific heats, and $\Gamma_{m}$ and $m_{m}$ are constants, $v=\rho^{-1}$ is phase specific volume and $v_{m}^{*}$ is a reference value for the specific volume of the metal phase. The pressure is given by:

$$
P_{m}=\frac{\widehat{e}_{m}}{v_{m}} G_{m}+f_{m}\left(v_{m}\right)
$$

where

$$
f_{m}\left(v_{m}\right)= \begin{cases}P_{H}-\frac{G_{m}}{v_{m}} e_{H}, & \frac{s_{m}-1}{s_{m}} v_{m}^{*}<v_{m}<v_{m}^{*} \\ \omega_{m}^{2}\left(\frac{1}{v_{m}}-\frac{1}{v_{m}^{*}}\right), & v_{m}>v_{m}^{*},\end{cases}
$$

and

$$
P_{H}=\left[\frac{\omega_{m}}{v_{m}^{*}-s_{m}\left(v_{m}^{*}-v_{i}\right)}\right]^{2}\left(v_{m}^{*}-v_{m}\right), \quad e_{H}=\frac{1}{2}\left[\frac{\omega_{m}\left(v_{m}^{*}-v_{m}\right)}{v_{m}^{*}-s_{m}\left(v_{m}^{*}-v_{m}\right)}\right]^{2} ;
$$

here, $s_{m}$ and $\omega_{m}$ are empirical constants. Finally, the gas phase is governed by the non-ideal, virial equation of state:

$$
\widehat{e}_{g}=\frac{P_{g}}{\left(\gamma_{g}-1\right) \rho_{g}\left(1+b_{g} \rho_{g}\right)},
$$

where $b_{g}$ is the virial coefficient.

Finally, a note on the detonation structure resolution is in order. As a means of verification, the simulations performed by Schwendeman et al. in [39] for a neat explosive, including the different forms of the interphase source terms used, were replicated. However, a uniform spatial grid was used here instead of the adaptive mesh refinement (AMR) technique applied in [39]. The results shown here are also compared to an algebraic end state analysis; details of these simulations and the end-state analysis are provided in [11]. It is evident from the simulations that the relaxation layers behind the detonation front are under-resolved. Additional numerical experiments also indicate that these regions continue to be under-resolved even for significantly more refined grids, which suggests that this is an inherent limitation of using uniformly-spaced grids to predict detonation phenomena. However, as shown in [11], the predictions approach the analytical end state at the end of the reaction zone. Consequently, the predictions within the remainder of the reaction zone are not likely to vary substantially with increasing resolution. Predictions are first shown for inert metal in Section 4.1, followed by an overview of additional model modifications and results for reactive metal in Section 4.2.

\subsection{Inert Aluminum}

Figure 3 shows spatial profiles behind the detonation front for a metal particle size $d_{m}=100 \mathrm{~nm}$ and metal concentration $\lambda_{m}^{0}=25 \%$, where the position $\xi=0$ is the location of the detonation front. Since the drag coefficient is proportional to $d^{-2}$, the metal and gas-phase velocities in Fig. 3(b) rapidly equilibrate, while the explosive component velocity relaxes over a longer length scale. As shown in Fig. 3(a), the solid-phase shocks precede the gaseous detonation, resulting in the thin compaction region indicated in Fig. 3, where the explosive volume fraction slightly increases prior to the arrival of the detonation front. Fig. 3(d) shows the existence of shocks in each phase, since the Mach number $\mathrm{Ma}_{k}=\left|u_{k}-D\right| / c_{k}<1$ 


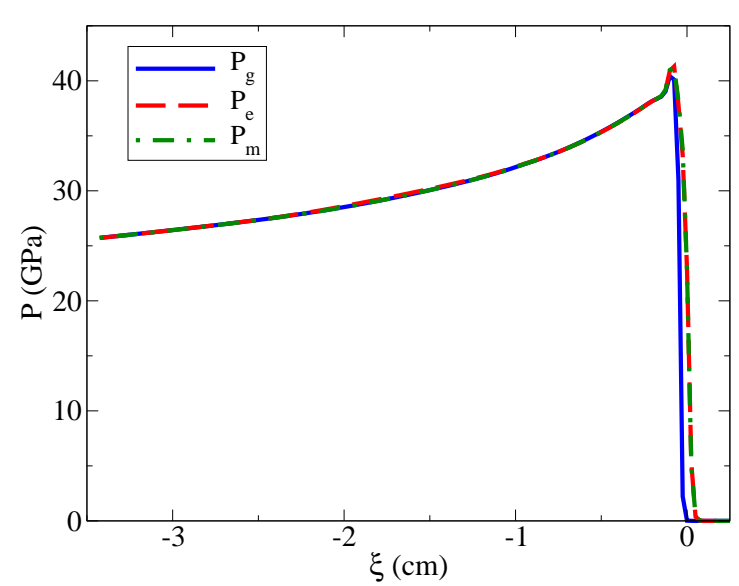

(a)

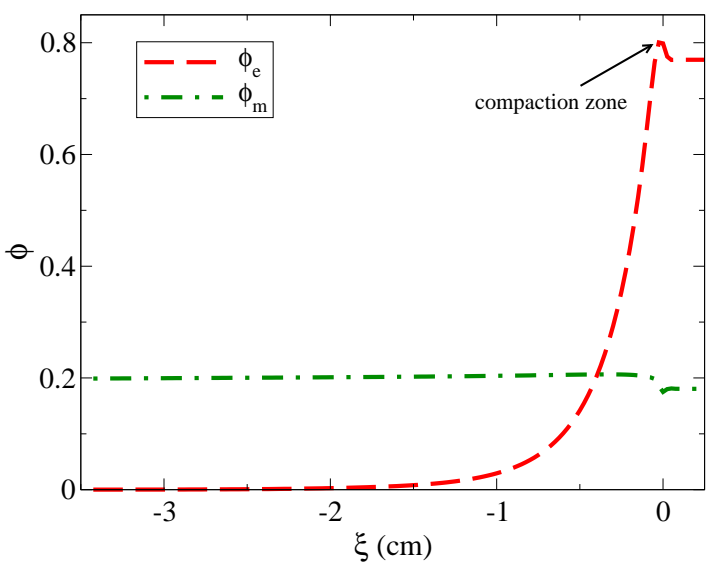

(c)

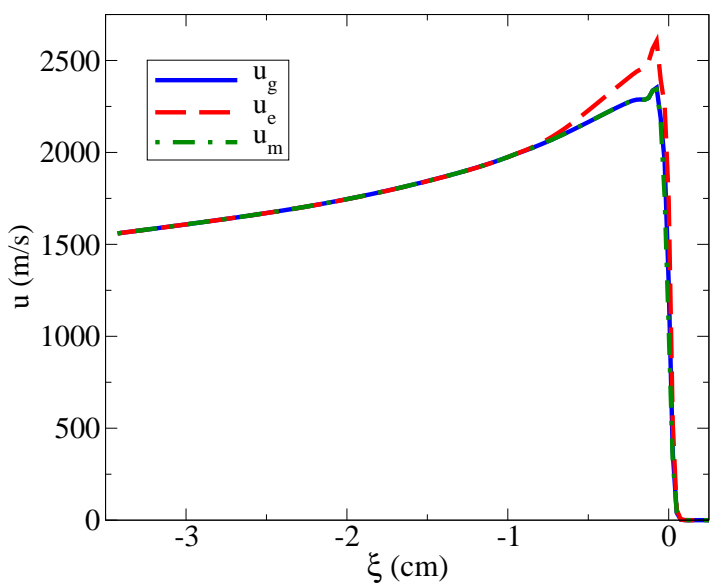

(b)

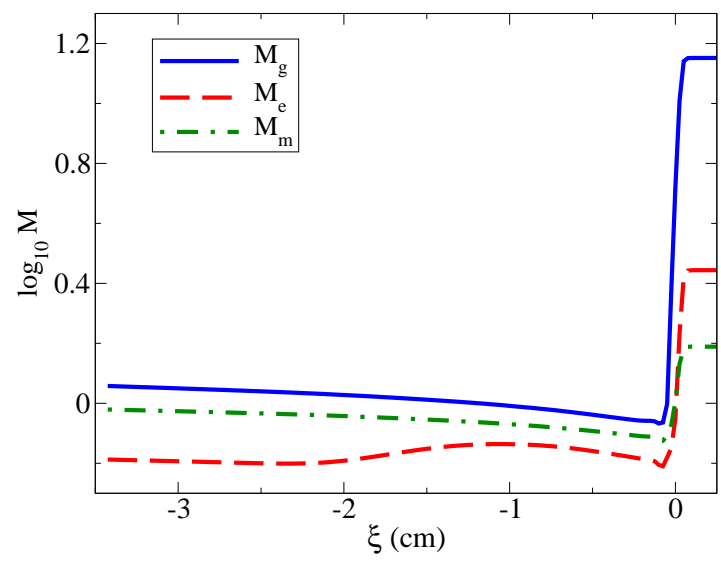

(d)

Figure 3: Steady Detonation Profiles for Phase (a) Pressure, (b) Velocity, (c) Volume Fraction, and (d) Mach Numbers for Inert Metal

behind the leading waves in each phase, where $D$ is the detonation speed and $c_{k}$ is phase sound speed for $k=e, m, g$. While the explosive phase remains subsonic, the metal phase approaches the sonic locus, and the gas phase becomes supersonic behind the reaction zone, indicating the prediction of a weak detonation.

Figure 4 shows the corresponding energetic response, where the phase temperature fields are given Fig. 4(a), with the temperature change decompositions shown in Figs. 4(b)-(d). In the latter plots the temperature changes due to each energetic process are normalized by the Chapman-Jouguet (CJ) temperature $T_{C J}$. The heat transfer coefficient is also proportional to $d^{-2}$, resulting in much more rapid thermal equilibration for the metal phase than the explosive component, as shown in Fig. 4(a). The solid explosive temperature change decomposition in Fig. 4(b) shows that compression is the dominant heating mechanism in the explosive immediately behind the detonation wave, while the influence of heat transfer from the gas increases within the explosive reaction zone, and subsequent expansion of the solid explosive in this region lowers its temperature. Compaction and drag have a negligible effect, as indicated in Fig. 4(b) and its inset, though it should be noted that additional dissipation is produced within the reaction zone. This is due to a small degree of phase pressure disequilibrium shown in Fig. 3(a) that originates during the early-time transition to detonation [39]. Similar behavior is predicted in the metal phase in Fig. 4(c), though the effects of compressive heating are more pronounced within the reaction zone. As in the neat explosive case, Fig. 4(d) shows that compression and drag act to increase gas temperatures, while heat transfer to the solid phases decreases temperature, and the combustion reaction further decreases gas temperature due to the production of additional gas. 


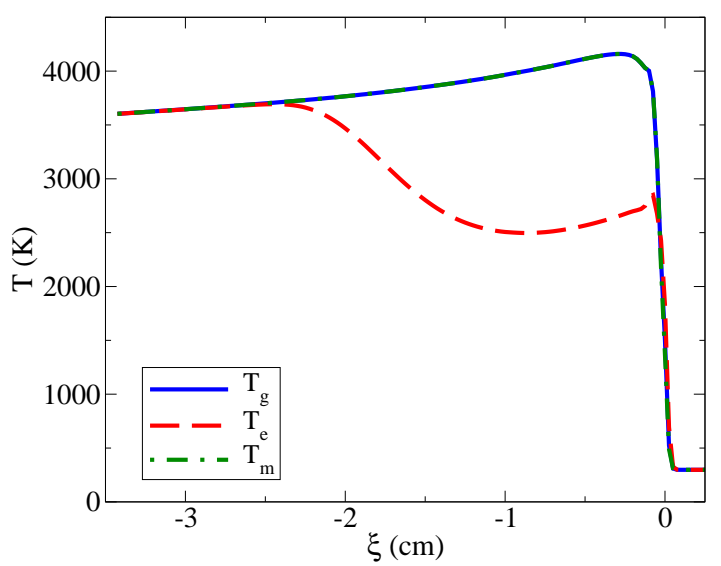

(a)

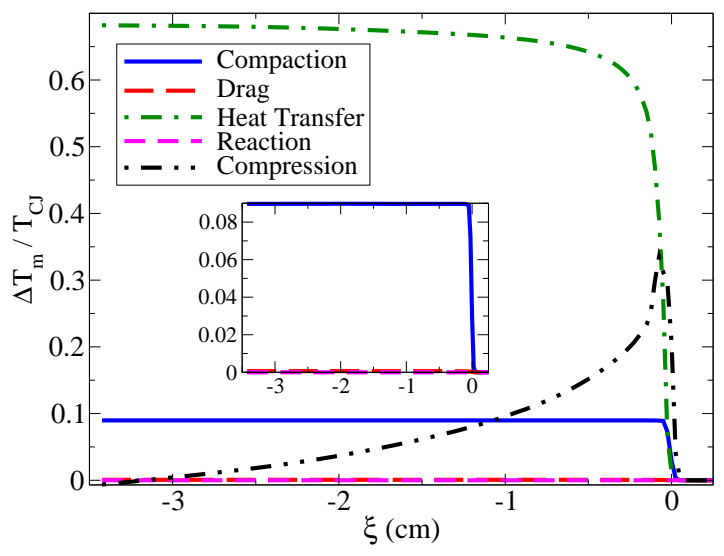

(c)

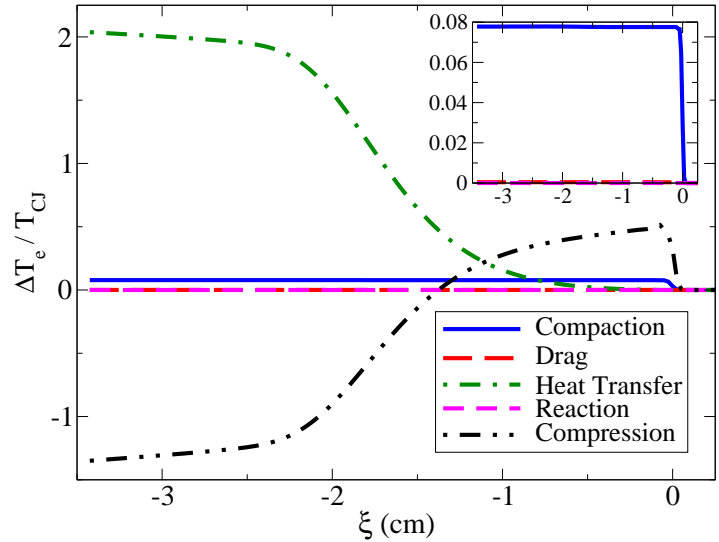

(b)

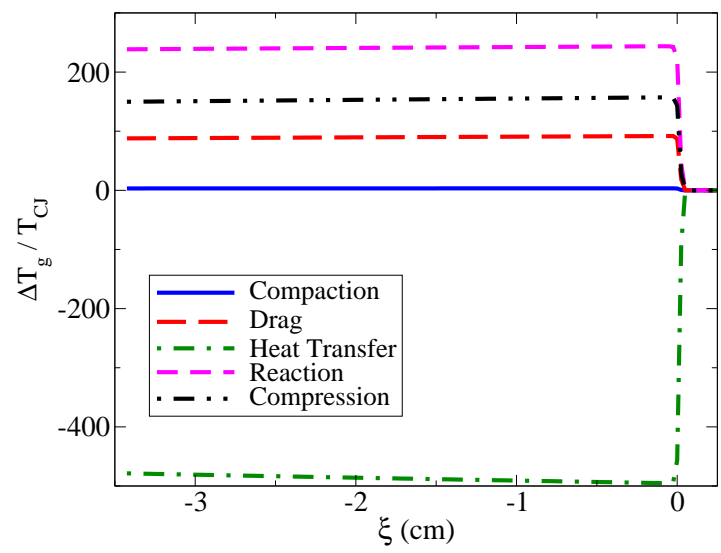

(d)

Figure 4: Steady Detonation Profiles for Phase (a) Temperature and Normalized Temperature Change Decomposition for (b) Explosive, (c) Inert Metal, and (d) Gas Phases

\subsection{Reactive Aluminum}

Here, the metal phase is allowed to react chemically with the gaseous explosive combustion products. The aluminum particles are taken to consist of an inner core of pure metal, initially coated with an oxide layer of thickness $\delta_{m}^{0}$. Unlike the detailed metal reaction pathways and phase change processes considered in [36], the thermodynamics and oxidation mechanics of the aluminum component are simplified here, both for modeling tractability and isolating the effects of metal reactions on the system energetics; details of this procedure are provided in [11]. The aluminum oxidation results in metal phase self-heating. Unlike the mass transfer mechanism associated with the combustion of high explosives, the most significant mode of energy release from the metal phase to the gas occurs due to convective heat transfer. Therefore, the modification of the internal energy of the metal phase formulated in [17] is adopted:

$$
e_{m}=e_{m}\left(\rho_{m}, P_{m}, \phi_{m}, \Pi_{m}\right)=\widehat{e}_{m}\left(\rho_{m}, P_{m}\right)+B_{m}\left(\phi_{m}\right)-\Pi_{m} q_{c h, m},
$$

where $0 \leq \Pi_{m} \leq 1$ is an oxidation reaction progress variable and $q_{c h, m}$ is the metal-phase heat of reaction. This modified internal energy formulation results in the inclusion of an additional term in the mixture entropy inequality, given by $\phi_{m} \rho_{m} q_{c h, m} d \Pi_{m} / d t_{m}$. Thus, this extra contribution to the mixture entropy will be non-negative if $d \Pi_{m} / d t_{m} \geq 0$ identically. The introduction of the oxidation reaction to the metal equation of state results in an additional temperature change decomposition variable $\mathcal{T}_{\text {ox,m }}$ that must be evolved separately, and increases the number of model equations accordingly. This equation has the same form as those given in Eq. (35), with the temperature change decomposition source term associated with metal oxidation given by:

$$
S_{o x, m}=\frac{1}{c_{v m}}\left[\phi_{m} \rho_{m} q_{c h, m}\left(\frac{1}{1-\delta_{m}^{0} / r_{m}}\right) \frac{d}{d t_{m}}\left(\frac{\delta_{m}}{r_{m}}\right)\right],
$$


where $r_{m}$ is the aluminum particle radius.

It is reasonable to expect that $\Pi_{m}$ is related to the oxide layer thickness $\delta_{m}$. Here, an expression for the growth of the oxide layer is used, which is similar to the Arrhenius-type relation provided in [42]; here, oxidation occurs until the oxide thickness is equal to $r_{m}$ :

$$
\frac{d \Pi_{m}}{d t_{m}}= \begin{cases}\frac{\sigma_{m g} C_{o x}}{\delta_{m}} \exp \left(-\frac{E_{a}}{R_{u} T_{m}}\right), & 0<\delta_{m}<r_{m}, \\ 0, & \text { otherwise }\end{cases}
$$

where $\sigma_{m g}$ is a reaction prefactor, $C_{o x}$ is the concentration of oxidizer in the gas phase, $E_{a}$ is the activation energy and $R_{u}$ is the universal gas constant. Initial values of the oxide thickness at ambient conditions must be selected such that the mass of this layer does not exceed a certain fraction of the total particle mass $\epsilon_{m}$. For aluminum, $\epsilon_{m}=2 \%$ [14]; this results in an inequality for the initial oxide layer thickness:

$$
0<\frac{\delta_{m}^{0}}{r_{m}}<1-\left[1+\frac{\rho_{m}^{0}}{\rho_{o x}^{0}}\left(\frac{\epsilon_{m}}{1-\epsilon_{m}}\right)\right]^{-1 / 3},
$$

where $\rho_{o x}^{0}$ is the ambient density of the oxide layer. In the absence of more accurate expressions, a simple linear relationship between $\delta_{m}$ and $\Pi_{m}$ is assumed, which satisfies the limiting cases, i.e., $\Pi_{m}=0$ when $\delta_{m}=\delta_{m}^{0}$, and $\Pi_{m}=1$ when $\delta_{m}=r_{m}$. This function is given by $\Pi_{m}=\left(\delta_{m}-\delta_{m}^{0}\right) /\left(r_{m}-\delta_{m}^{0}\right)$. The Lagrangian oxidation rate equation (85) can be expressed in the divergence form utilized in the other model equations in a straightforward manner. This is given by:

$$
\begin{gathered}
\frac{\partial}{\partial t}\left(\phi_{m} \rho_{m} \delta_{m}^{*}\right)+\frac{\partial}{\partial x}\left(\phi_{m} \rho_{m} u_{m} \delta_{m}^{*}\right)=F_{m} ; \\
F_{m}=\frac{\phi_{m} \rho_{m} \sigma_{m g} C_{o x}}{r_{m}^{2} \delta_{m}^{*}} \exp \left(-\frac{E_{a}}{R_{u} T_{m}}\right)+\mathcal{C}_{m} \delta_{m}^{*}
\end{gathered}
$$

for $0<\delta_{m}^{*}<1$, and $F_{m}=0$ otherwise, where $\delta_{m}^{*}=\delta_{m} / r_{m}$. Therefore, the model presented in Section 2 is supplemented by Eq. (88), where $\Pi_{m}$ is then obtained from the linear expression.

Here, $\phi_{s}^{0}=95 \%$ and the initial metal mass fraction $\lambda_{m}^{0}$ is held constant at $15 \%$; values for the parameters used in Eq. (85) are given in [16]. Figure 5 shows the energetic response for $d_{e}=100 \mu \mathrm{m}$ and $d_{m}=100 \mathrm{~nm}$. The position $\xi$ is again measured relative to the detonation front; however, the spatial domain is expanded here, where $\xi$ is normalized using the length of the explosive reaction zone $\Delta \xi_{r z}$ to distinguish between energetic processes occurring both within the reaction zone and the subsequent expansion wave. Thus, the explosive reaction zone corresponds to $-1 \leq \xi / \Delta \xi \leq 0$, and the Taylor wave corresponds to $\xi / \Delta \xi<-1$.

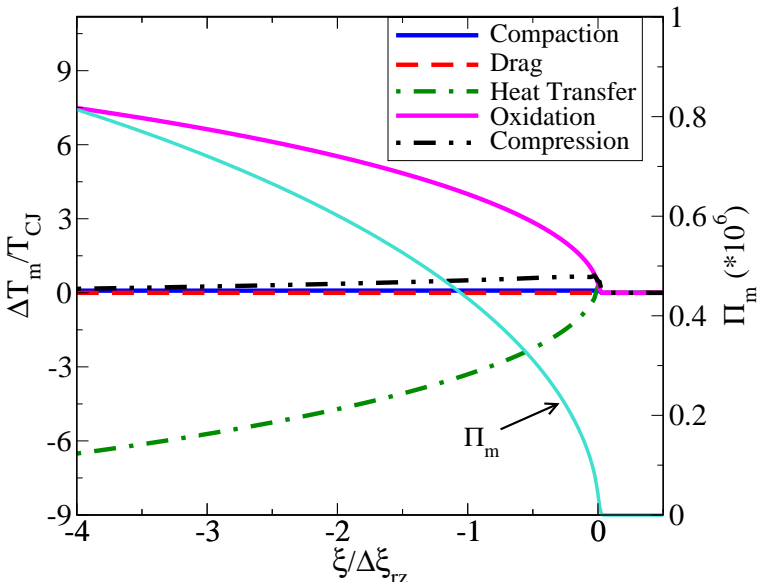

(a)

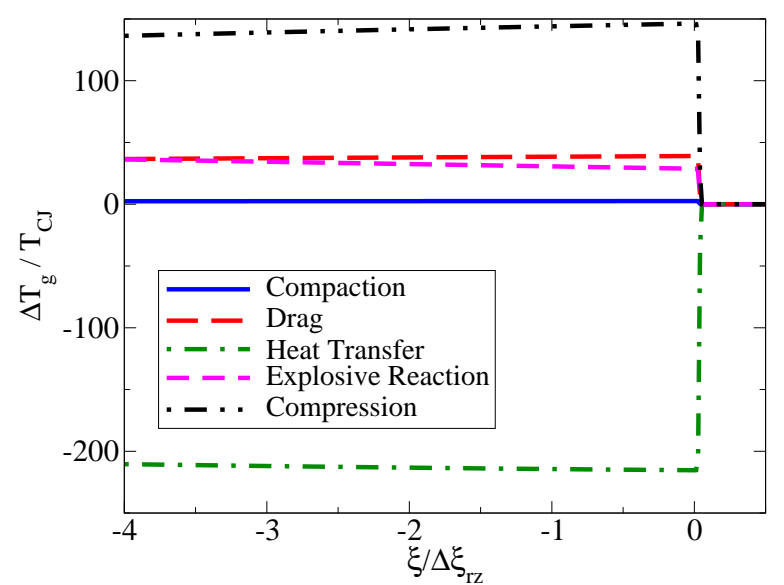

(b)

Figure 5: Detonation Profiles for Normalized Temperature Change Decomposition for (a) Reactive Metal and (b) Gas Phases

Here, the role of internal metal heating due to oxidation is evident; as shown in Fig. 5(a), its contribu- 
tion to metal energetics becomes dominant immediately behind the detonation front. The negative heat transfer contribution from the metal phase confirms that, unlike the inert metal, the reactive aluminum serves as a heat source for the explosive component. Consequently, if internal exothermic metal heating occurs rapidly within the explosive reaction zone, heat transfer from the metal could be coupled to the wave front, resulting in an accelerated detonation relative to the inert case [11]. This is further suggested by the dominance of compressive heating in the gas phase in Fig. 5(b) due to higher post-detonation pressures.

It is noted that the change from the $25 \%$ metal content used in Section 4.1 to the $15 \%$ content used here was motivated by the behavior of the metal reaction progress variable $\Pi_{m}$ within the computational domain. As shown in Fig. 5, at $15 \%$ there is a clear transition from rapidly rising, yet smaller, values of $\Pi_{m}$ within the reaction zone $(-1 \leq \xi / \Delta \xi \leq 0)$ to significantly larger values outside this region within the Taylor wave. Together with the oxidation and heat transfer profiles in Fig. 5(a), this indicates a low amount of oxidative heating within the reaction zone followed by a significantly larger amount outside the zone. At 25\%, preliminary results indicated that the large amount of metal results in nearly instant oxidation immediately behind the detonation front, and the transition mode present at $15 \%$ metal does not occur. Consequently, the lower metal content more clearly illustrates a shift in the metal energetics from the endothermic combustion-gas heat transfer mode to exothermic oxidative heating.

\section{Conclusions}

In this work a hyperbolic multiphase model was formulated to describe the engineering-scale behavior of energetic mixtures containing an arbitrary number of reactive solid components. A mathematically rigorous analysis, based on the mixture entropy inequality, was performed to identify physically permissible interphase exchange processes. This analysis involved the specification of partitioning functions used to apportion energy dissipation between each phase; although many of these functions were previously defined for two-phase reactive systems [5] and inert multiphase systems with $N>1$ [18], this work introduces several new dissipation partitioning functions associated with general chemical reactions of the type $A \rightarrow B$, which must be specified. Furthermore, selection criteria for these new functions were established that enforces compliance with the strong form of the Second Law, while imposing no constraints a priori on the functional form of the chemical reaction laws. In the special case of combustion reactions only, this general framework exhibits the proper limiting behavior by reducing to an $N$-phase extension of the two-phase model in [5], and further reduces to the inert multiphase model in [18] when no reactions occur.

Instantaneous energy transport and dissipation rates are commonly used in the literature to identify significant processes occurring at a fixed instant in time within the spatial domain [18, 39]; however, these rates are unable to compute the cumulative effect of these energetic processes over time, where the duration of a high-rate transient event may be brief relative to a steady process occurring over a longer time scale. The model developed in this work was therefore supplemented with additional evolution equations describing the relative contributions of each energetic process to the total changes in phase temperatures over the entire simulation time. This was accomplished by decomposing the total temperature change into multiple contributions from different transport and dissipation processes, with sources obtained from the phase entropy evolution equations. These decompositions are not unique, and may be further subdivided into additional processes depending on the application.

The model was then used to evolve steady detonation waves in a metalized explosive for both inert and reactive metal components to illustrate the effects of metal oxidation on the resulting steady detonation profiles. The rapid exothermic metal heating and resulting heat transfer for small metal particle sizes suggest that this energy release could be coupled to the explosive reaction zone, thereby significantly affecting detonation speed and strength. The novel reactive modeling capabilities developed here therefore provide a basis for predicting the sensitivity of metalized explosive performance metrics to mixture properties, as well as determining the most significant energetic processes which contribute to explosive performance.

\section{Acknowledgments}

The authors gratefully acknowledge support this research provided by the Defense Threat Reduction Agency (DTRA) under sponsor award number HDTRA1-10-1-0018. This work was also supported by the National Science Foundation Integrative Graduate Education and Research Traineeship on Computational Fluid Dynamics at Louisiana State University, grant number DGE-0504507. 


\section{Appendix A. Entropy Inequality Derivations}

From the solid equation of state,

$$
d e_{i}=d \widehat{e}_{i}+\frac{d B_{i}}{d \phi_{i}} d \phi_{i}
$$

and from the Gibbs relation,

$$
d \widehat{e}_{i}=T_{i} d \eta_{i}+\frac{P_{i}}{\rho_{i}^{2}} d \rho_{i}, \quad d \widehat{e}_{g}=T_{g} d \eta_{g}+\frac{P_{g}}{\rho_{g}^{2}} d \rho_{g},
$$

where $\eta_{i}$ and $\eta_{g}$ are the values of specific entropy for the $i$-th solid phase and gas phase, respectively. Thus,

$$
T_{i} \frac{d \eta_{i}}{d t_{i}}=\frac{d e_{i}}{d t_{i}}-\frac{P_{i}}{\rho_{i}^{2}} \frac{d \rho_{i}}{d t_{i}}-\frac{\beta_{i}}{\phi_{i} \rho_{i}} \frac{d \phi_{i}}{d t_{i}}
$$

similarly for the gas phase,

$$
T_{g} \frac{d \eta_{g}}{d t_{g}}=\frac{d e_{g}}{d t_{g}}-\frac{P_{g}}{\rho_{g}^{2}} \frac{d \rho_{g}}{d t_{g}}
$$

where $d / d t_{g}=\partial / \partial t+\mathbf{u}_{g} \cdot \nabla$.

The entropy evolution for each solid phases is first derived by obtaining Lagrangian rate expressions for $e_{i}, \rho_{i}$ and $\phi_{i}$. Together with the compaction equation (12), the continuity equation can be expressed as:

$$
\phi_{i} \frac{d \rho_{i}}{d t_{i}}+\phi_{i} \rho_{i} \nabla \cdot \mathbf{u}_{i}=-\rho_{i} \mathcal{F}_{i} .
$$

Since the evolution equation for $\phi_{i}$ is already given by Eq. (12), the final step is deriving an expression for $e_{i}$. The continuity equation may be substituted into the total energy equation to obtain:

$$
\phi_{i} \rho_{i} \frac{d E_{i}}{d t_{i}}=\nabla \cdot\left(\phi_{i} P_{i} \mathbf{u}_{i}\right)+\mathcal{E}_{i}-\mathcal{C}_{i} E_{i}
$$

The momentum equation can also be expressed in Lagrangian form using the continuity equation:

$$
\phi_{i} \rho_{i} \frac{d \mathbf{u}_{i}}{d t_{i}}=\nabla\left(\phi_{i} P_{i}\right)+\overrightarrow{\mathcal{M}}_{i}-\mathcal{C}_{i} \mathbf{u}_{i}
$$

The kinetic energy contribution to $E_{i}$ is obtained by taking the dot product of $\mathbf{u}_{i}$ and both sides of Eq. (A.7). Subtracting the resulting expression from Eq. (A.6) yields:

$$
\phi_{i} \rho_{i} \frac{d e_{i}}{d t_{i}}=-\phi_{i} P_{i} \nabla \cdot \mathbf{u}_{i}+\mathcal{E}_{i}-\overrightarrow{\mathcal{M}}_{i} \cdot \mathbf{u}_{i}-\mathcal{C}_{i} e_{i}+\mathcal{C}_{i} \frac{\left|\mathbf{u}_{i}\right|^{2}}{2}
$$

Substituting Eqs. (A.5), (12) and (A.8) into Eq. (22) gives:

$$
\phi_{i} \rho_{i} T_{i} \frac{d \eta_{i}}{d t_{i}}=\mathcal{E}_{i}-\overrightarrow{\mathcal{M}}_{i} \cdot \mathbf{u}_{i}-\mathcal{C}_{i}\left(e_{i}+\frac{\beta_{i}}{\rho_{i}}-\frac{\left|\mathbf{u}_{i}\right|^{2}}{2}\right)+\left(P_{i}-\beta_{i}\right) \mathcal{F}_{i}
$$

For the gas phase, the relation for $e_{g}$ is obtained in an identical manner:

$$
\phi_{g} \rho_{g} \frac{d e_{g}}{d t_{g}}=-\phi_{g} P_{g} \nabla \cdot \mathbf{u}_{g}+\mathcal{E}_{g}-\overrightarrow{\mathcal{M}}_{g} \cdot \mathbf{u}_{g}-\mathcal{C}_{g} e_{g}+\mathcal{C}_{g} \frac{\left|\mathbf{u}_{g}\right|^{2}}{2} .
$$

An evolution equation for $\rho_{g}$ is obtained in a somewhat different manner. The gas-phase continuity equation is expressed as:

$$
\frac{d}{d t_{g}}\left(\phi_{g} \rho_{g}\right)+\phi_{g} \rho_{g} \nabla \cdot \mathbf{u}_{g}=\mathcal{C}_{g}
$$

Recognizing that $d / d t_{i}=d / d t_{g}+\left(\mathbf{u}_{i}-\mathbf{u}_{g}\right) \cdot \nabla$, from Eq. (12) and the saturation constraint,

$$
\frac{d \phi_{g}}{d t_{g}}=-\sum_{j=1}^{M} \frac{d \phi_{j}}{d t_{g}}=-\sum_{j=1}^{M}\left[\mathcal{F}_{j}+\frac{\mathcal{C}_{j}}{\rho_{j}}-\left(\mathbf{u}_{j}-\mathbf{u}_{g}\right) \cdot \nabla \phi_{j}\right] .
$$


Subtracting Eq. (A.12) from Eq. (A.11) yields:

$$
\frac{d \rho_{g}}{d t_{g}}=\frac{1}{\phi_{g}} \sum_{j=1}^{M} \mathcal{C}_{g}\left(\frac{\rho_{g}}{\rho_{j}}-1\right)-\rho_{g} \nabla \cdot \mathbf{u}_{g}+\frac{\rho_{g}}{\phi_{g}} \sum_{j=1}^{M}\left[\mathcal{F}_{j}-\left(\mathbf{u}_{j}-\mathbf{u}_{g}\right) \cdot \nabla \phi_{j}\right] .
$$

Substituting Eqs. (A.10) and (A.12) into Eq. (23) gives:

$$
\begin{aligned}
\phi_{g} \rho_{g} T_{g} \frac{d \eta_{g}}{d t_{g}} & =\mathcal{E}_{g}-\overrightarrow{\mathcal{M}}_{g} \cdot \mathbf{u}_{g}-\mathcal{C}_{g}\left(e_{g}-\frac{\left|\mathbf{u}_{g}\right|^{2}}{2}\right)-\frac{P_{g}}{\rho_{g}} \sum_{j=1}^{M} \mathcal{C}_{j}\left(\frac{\rho_{g}}{\rho_{j}}-1\right) \\
& -P_{g} \sum_{j=1}^{M}\left[\mathcal{F}_{j}-\left(\mathbf{u}_{j}-\mathbf{u}_{g}\right) \cdot \nabla \phi_{j}\right] .
\end{aligned}
$$

\section{Appendix B. Gas-Solid and Solid-Solid Entropy Relations}

For quantities $z_{i}$ and $z_{g}$ (scalar or vector), which are functions of the $i$-th solid phase and gas phase properties, respectively, summation yields:

$$
\sum_{j=1}^{M} \sum_{n=1}^{M}\left(\frac{z_{j}}{T_{j}}-\frac{z_{g}}{T_{g}}\right) \mathcal{W}_{j n}=\sum_{j=1}^{M} \frac{z_{j}}{T_{j}} \sum_{n=1}^{M} \mathcal{W}_{j n}=\sum_{j=1}^{M-1} \sum_{n=j+1}^{M}\left(\frac{z_{j}}{T_{j}}-\frac{z_{n}}{T_{n}}\right) \mathcal{W}_{j n},
$$

where the implied multiplication indicates either a scalar or vector dot product. Consequently, Eq. (B.1) can be substituted within each term in Eq. (28), with the final term given by:

$$
\frac{P_{g}}{T_{g}} \sum_{j=1}^{M} \frac{1}{\rho_{j}} \sum_{n=1}^{M} \mathcal{C}_{j n}=\frac{P_{g}}{T_{g}} \sum_{j=1}^{M-1} \sum_{n=j+1}^{M}\left(\frac{1}{\rho_{j}}-\frac{1}{\rho_{n}}\right) \mathcal{C}_{j n} .
$$

The mixture entropy inequality given by Eq. (28) can be cast in terms of this decomposition:

$$
\sum_{j=1}^{M} \mathcal{A}_{j}+\sum_{j=1}^{M-1} \sum_{n=j+1}^{M} \mathcal{A}_{j n} \geq 0
$$

where

$$
\begin{aligned}
& \mathcal{A}_{j}=\left\{\left(\frac{1}{T_{j}}-\frac{1}{T_{g}}\right) \mathcal{E}_{j g}-\left(\frac{\mathbf{u}_{j}}{T_{j}}-\frac{\mathbf{u}_{g}}{T_{g}}\right) \cdot \overrightarrow{\mathcal{M}}_{j g}+\left(\frac{P_{j}-\beta_{j}}{T_{j}}-\frac{P_{g}}{T_{g}}\right) \mathcal{F}_{i g}-\left[\left(\frac{1}{T_{j}}\right)\left(e_{j}+\frac{\beta_{j}}{\rho_{j}}-T_{j} \eta_{j}\right)\right.\right. \\
&\left.\left.-\left(\frac{1}{T_{g}}\right)\left(e_{g}+P_{g}\left(\frac{1}{\rho_{g}}-\frac{1}{\rho_{j}}\right)-T_{g} \eta_{g}\right)\right] \mathcal{C}_{j g}+\left(\frac{\left|\mathbf{u}_{j}\right|^{2}}{2 T_{j}}-\frac{\left|\mathbf{u}_{g}\right|^{2}}{2 T_{g}}\right) \mathcal{C}_{i g}+\frac{P_{g}}{T_{g}}\left(\mathbf{u}_{j}-\mathbf{u}_{g}\right) \cdot \nabla \phi_{j}\right\} \\
& \mathcal{A}_{j n}=\left\{\left(\frac{1}{T_{j}}-\frac{1}{T_{n}}\right) \mathcal{E}_{j n}-\left(\frac{\mathbf{u}_{j}}{T_{j}}-\frac{\mathbf{u}_{n}}{T_{n}}\right) \cdot \overrightarrow{\mathcal{M}}_{i n}+\left(\frac{P_{j}-\beta_{j}}{T_{j}}-\frac{P_{n}-\beta_{n}}{T_{n}}\right) \mathcal{F}_{i n}\right. \\
&- {\left[\left(\frac{1}{T_{j}}\right)\left(e_{j}+\frac{\beta_{j}+P_{g} T_{j} / T_{g}}{\rho_{j}}-T_{j} \eta_{j}\right)\right.} \\
&-\left.\left.\left(\frac{1}{T_{n}}\right)\left(e_{n}+\frac{\beta_{n}+P_{g} T_{n} / T_{g}}{\rho_{n}}-T_{n} \eta_{n}\right)\right] \mathcal{C}_{j n}+\left(\frac{\left|\mathbf{u}_{j}\right|^{2}}{2 T_{j}}-\frac{\left|\mathbf{u}_{n}\right|^{2}}{2 T_{n}}\right) \mathcal{C}_{j n}\right\}
\end{aligned}
$$

The entropy evolution equations, which are used to evaluate the sources $\mathbf{S}_{p}$ for the temperature change decomposition equations, are therefore given by:

$$
\begin{aligned}
\phi_{i} \rho_{i} T_{i} \frac{d \eta_{i}}{d t_{i}} & =\mathcal{E}_{i g}-\overrightarrow{\mathcal{M}}_{i g} \cdot \mathbf{u}_{i}-\mathcal{C}_{i g}\left(e_{i}+\frac{\beta_{i}}{\rho_{i}}-\frac{\left|\mathbf{u}_{i}\right|^{2}}{2}\right)+\left(P_{i}-\beta_{i}\right) \mathcal{F}_{i g}+\sum_{n=1}^{M}\left[\mathcal{E}_{i n}-\overrightarrow{\mathcal{M}}_{i n} \cdot \mathbf{u}_{i}\right. \\
& \left.-\mathcal{C}_{i n}\left(e_{i}+\frac{\beta_{i}}{\rho_{i}}-\frac{\left|\mathbf{u}_{i}\right|^{2}}{2}\right)+\left(P_{i}-\beta_{i}\right) \mathcal{F}_{i n}\right]
\end{aligned}
$$


for $i=1,2, \ldots, M$, and

$$
\begin{aligned}
& \phi_{g} \rho_{g} T_{g} \frac{d \eta_{g}}{d t_{g}}=-\sum_{j=1}^{M}\left\{\mathcal{E}_{j g}-\overrightarrow{\mathcal{M}}_{j g} \cdot \mathbf{u}_{g}-\mathcal{C}_{j g}\left[e_{g}-\frac{\left|\mathbf{u}_{g}\right|^{2}}{2}\right.\right. \\
& \left.\left.-P_{g}\left(\frac{1}{\rho_{j}}-\frac{1}{\rho_{g}}\right)\right]+P_{g}\left[\mathcal{F}_{j g}-\left(\mathbf{u}_{j}-\mathbf{u}_{g}\right) \cdot \nabla \phi_{j}\right]\right\}-P_{g} \sum_{n=j+1}^{M} \sum_{j=1}^{M-1}\left(\frac{1}{\rho_{j}}-\frac{1}{\rho_{n}}\right) \mathcal{C}_{j n} .
\end{aligned}
$$

Equation (27) is then used to obtain the mixture entropy inequality in terms of the decomposed gas-solid and solid-solid interactions.

\section{References}

[1] Baer, M.R., 2002. Modeling heterogeneous energetic materials at the mesoscale. Thermochimica Acta 384, 351-367.

[2] Baer, M.R., 2007. Shock Wave Science and Technology Reference Library. Springer Berlin Heidelberg. volume 2. chapter Mesoscale Modeling of Shocks in Heterogeneous Reactive Materials. pp. 321-356.

[3] Baer, M.R., Nunziato, J.W., 1986. A two-phase mixture theory for the deflagration-to-detonation transition (DDT) in reactive granular materials. Int. J. Multiphas. Flow 12, 861-889.

[4] Baer, M.R., Trott, W.M., 2002. Mesoscale descriptions of shock-loaded heterogeneous porous materials, in: AIP Conference Proceedings, pp. 713-716.

[5] Bdzil, J.B., Menikoff, R., Son, S.F., Kapila, A.K., Stewart, D.S., 1999. Two-phase modeling of deflagration-todetonation transition in granular materials: A critical examination of modeling issues. Phys. Fluids 11, 378-402.

[6] Borg, J.P., Vogler, T.J., 2008. Mesoscale calculations of the dynamic behavior of a granular ceramic. International Journal of Solids and Structures 45, 1676-1696.

[7] Brousseau, P., Anderson, C.J., 2002. Nanometric aluminum in explosives. Propell. Explos. Pyrot. 27, $300-306$.

[8] Chinnayya, A., Daniel, E., Saurel, R., 2004. Modelling detonation waves in heterogeneous energetic materials. J. Comput. Phys. 196, 490-538.

[9] Coquel, F., El Amine, K., Godlewski, E., Perthame, B., Rascle, P., 1997. A numerical method using upwind schemes for the resolution of two-phase flows. J. Comput. Phys. 136, 272-288.

[10] Coutinho, S.A., Elves, J.J.N., Martignoni, W.P., Mortignoni, W.P., Mori, M., 2004. Modelling and simulation of gas-solid flows. Adv. Fluid Mech. Ser. 37, 199-208.

[11] Crochet, M.W., 2013. Modeling, Numerical Analysis, and Predictions for the Detonation of Multi-Component Energetic Solids. Ph.D. thesis. Louisiana State University.

[12] Crochet, M.W., Gonthier, K.A., 2013. Numerical investigation of a modified family of centered schemes applied to multiphase equations with nonconservative sources. J. Comp. Phys. 255, 266-292.

[13] Fedorov, A.V., Kharlamova, Y.V., 2003. Ignition of an aluminum particle. Combust. Explo. Shock+ 39, 544-547.

[14] Fedorov, A.V., Khmel, T.A., 2012. Characteristics and criteria of ignition of suspensions of aluminum particles in detonation processes. Combust. Explo. Shock+ 48, 191-202.

[15] Gogulya, M.F., Makhov, M.N., Brazhnikov, M.A., Dolgoborodov, A.Y., Arkhipov, V.I., Zhigach, A.N., Leipunskii, I.O., Kuskov, M.L., 2008. Explosive characteristics of aluminized HMX-based nanocomposites. Combust. Explo. Shock+ 44, 198-212.

[16] Gogulya, M.F., Makhov, M.N., Dolgoborodov, A.Y., Brazhnikov, M.A., Arkhipov, V.I., Shchetinin, V.G., 2004. Mechanical sensitivity and detonation parameters of aluminized explosives. Combust. Explo. Shock+ 40, 445-457.

[17] Gonthier, K.A., 2003. Modeling and analysis of reactive compaction for granular energetic solids. Combust. Sci. Technol. 175, 1679-1709.

[18] Gonthier, K.A., Rumchik, C.G., 2007. A Multiphase Continuum Theory for Shock Loading of Metalized Explosive. Technical Report ME-TS1-09. Louisiana State University.

[19] Grishkin, A.M., Dubnov, L.V., Davidov, V.Y., Levshina, Y.A., Mikhallova, T.N., 1993. Effect of powdered aluminum additives on the detonation parameters of high explosives. Combust. Explo. Shock+ 29, 239-241.

[20] Hamdan, M.H., Siyyam, H.I., 2009. On the flow of a dusty gas with constant number density through granular porous media. Appl. Math. Comput. 209, 339-345.

[21] Howard, W.M., Fried, L.E., Souers, P.C., 2000. Modeling of non-ideal aluminized explosives, in: AIP Conf. Proc., pp. 389-392.

[22] Kosinski, P., 2008. Numerical investigation of explosion suppression by inert particles in straight ducts. J. Hazard. Mater. 154, 981-991.

[23] Krishenik, P.M., Rumanov, E.N., Shkadinskii, K.G., 1994. Modeling of combustion wave propagation in a carbon dust/gas mixture. Combust. Flame 99, 713-722.

[24] Lee, K.Y., Kennedy, J.E., Asay, B.W., Son, S.F., Martin, E.S., 2004. Preparation and characterization of fine particle NTO and its formulation with AL nanopowders, in: AIP Conf. Proc., pp. 855-858.

[25] LeFrancois, A., Baudin, G., Le Gallic, C., Boyce, P., Coudoing, J.P., 2002. Nanometric aluminum powder influence on the detonation efficiency of explosives, in: Det. Symp., San Diego, CA.

[26] Lu, H., Gidaspow, D., Bouillard, J., Liu, W., 2003. Hydrodynamic simulation of gas-solid flow in a riser using kinetic theory of granular flow. Chem. Eng. J. 95, 1-13.

[27] Lu, H., Zhao, Y., Shen, Z., Ding, J., Jiying, J., 2006. Numerical simulations of gas-solid flow in tapered risers. Powder Technol. 169, 89-98.

[28] McGrath II, T.P., 2008. Numerical Modeling of Multiphase Explosions. Ph.D. thesis. University of Maryland.

[29] Medvedev, A.E., Fedorov, A.V., Fomin, V.M., 1984. Description of ignition and combustion of gas mixtures with solid particles by methods of the mechanics of continuous media. Combust. Explo. Shock+ 20, 127-133. 
[30] Muravyev, N., Frolov, Y., Pivkina, A., Monogarov, K., Ordzhonikidze, O., 2010. Influence of particle size and mixing technology on combustion of HMX/Al compositions. Propell. Explos. Pyrot. 35, 226-232.

[31] Orth-Farrell, L., Krier, H., 2000. Simulation of detonation in high explosives with aluminum particles. Combust. Sci. Technol. 161, 69-88.

[32] Panchadhara, R., Gonthier, K.A., 2011. Mesoscale analysis of volumetric and surface dissipation in granular explosive induced by uniaxial deformation waves. Shock Waves 21, 43-61.

[33] Petitpas, F., Saurel, R., Franquet, E., Chinnayya, A., 2009. Modelling detonation waves in condensed energetic multiphase CJ conditions and multidimensional computations. Shock Waves 19, 377-401.

[34] Powers, J.M., 2004. Two-phase viscous modeling of compaction of granular materials. Physics of Fluids 16, $2975-2990$.

[35] R., S., Lemetayer, O., 2001. A multiphase model for compressible flows with interfaces, shocks, detonation waves and cavitation. J. Fluid Mech. 431, 239-271.

[36] Ruggirello, K.P., DesJardin, P.E., Baer, M.R., Kaneshige, M.J., Hertel, E.S., 2012. A reaction progress variable modeling approach for non-ideal multiphase explosives. Int. J. Multiphas. Flow 42, 128-151.

[37] Saurel, R., Abgrall, R., 1999. A multiphase Godunov method for compressible multifluid and multiphase flows. J. Comput. Phys. 150, 425-467.

[38] Saurel, R., Favrie, N., Petitpas, F., Lallemand, M.H., Gavrilyuk, S.L., 2010. Modelling dynamic and irreversible powder compaction. J. Fluid Mech. 664, 348-396.

[39] Schwendeman, D.W., Wahle, C.W., Kapila, A.K., 2008. A study of detonation evolution and structure for a model of compressible two-phase flow. Combust. Theor. Model. 12, 159-204.

[40] Sheng, J., Otuonye, F., 1988. Dust transport during coal mine explosion, in: US Mine Vent. Sym., pp. 540-548.

[41] Teselkin, V.A., 2008. Influence of the particle size of the components on the mechanical sensitivity of metallized high explosives. Russ. J. Phys. Chem. B 2, 609-618.

[42] Trunov, M.A., Schoenitz, M., Dreizin, E.L., 2005. Ignition of aluminum powders under different experimental conditions. Propell. Explos. Pyrot. 30, 36-43.

[43] van Wachem, B.G.M., Schouten, J.C., van den Bleek, C.M., Krishna, R., Sinclair, J.L., 2001. Comparative analysis of CFD models of dense gas-solid systems. AIChE J. 47, 1035-1051.

[44] Wang, W., Li, Y., 2001. Hydrodynamic simulation of fluidization by using a modified kinetic theory. Ind. Eng. Chem. Res. 40, 5066-5073.

[45] Webb, S.W., 1998. Gas-phase diffusion in porous media-evaluation of an advective-dispersive formulation and the dusty-gas model for binary mixtures. J. Porous Media 1, 187-199.

[46] Xu, S., Stewart, D.S., 1997. Deflagration-to-detonation transition in porous energetic materials: A comparative model study. J. Eng. Math. 31, 143-172.

[47] Zhang, F. (Ed.), 2009. Shock Wave Science and Technology Reference Library. Springer-Verlag. volume 4 . chapter 4.

[48] Zhang, F., Frost, D.L., Thibault, P.A., Murray, S.B., 2001. Explosive dispersal of solid particles. Shock Waves 10, 431-443.

[49] Zhong, S., Teodorczyk, A., Deng, X., Dang, J., 2002. Modeling and simulation of coal dust explosions. J. Phys. IV 12, Pr7/141-Pr7/147. 